\title{
Experimental Demonstration of the Relational Character of Time in Natural Processes; Concept of Time in the Relational World
}

\author{
A. Titov
}

\begin{abstract}
Using new experimental techniques, studies of thermal evolution processes are performed in systems, which are beyond the description of theoretical physics. Evolution process is characterized by braking symmetries in time and in space and by violations of conservation laws of energy and momentum. It is shown experimentally that the superposition principle is not valid for electromagnetic (EM) fields interacting with material objects; the Poynting vector of the external field is the source of changes in the properties and structure of the irradiated object; the thermal hysteresis loop presents a record of the process in two locations. The interaction of material objects through common EM field, which is carrying the contributions of the hysteresis effects in the parts of each interacting object, can be described only by the concept of a process that is characterized by its dependence on the huge number of correlated influence factors. The flux of Poynting vector of common EM field through boundary surface of the irradiated object is the reason for the development of the thermal process inside it, thus realizing the interrelation of one to all in the Leibniz relational World. Defining the concept of time as a reference process in experiments, in which the records of the states of one of the process are performed in terms of the states of the reference process in some material detector, we obtain the concept of time as a natural development of the basic principles of $G$. W. Leibniz, E. Mach, A. Einstein, W. Pauli, D. Kleppner.
\end{abstract}

Index Terms-evolution process, hysteresis, symmetry braking..

\section{INTRODUCTION}

Time and space are the fundamental concepts of theoretical physics, which define the relationship of physics to philosophy and determine its position among natural sciences. The concept of time (which is of primary importance for the subject of this paper) evolved gradually with epochs. It reflected the two main trends in the development of theoretical physics. The mainstream approach is based on the principles, which were laid down by I. Newton in his book "Mathematical Principles of Natural Philosophy" [1] and which L. Smolin in his book [2] called as Newtonian paradigm. The divide in the views of theorists relies on the way a person understands what a law of physics is. "According to the dominant view, everything that happens in the universe is determined by a law, which dictates

Alexandre Titov, Physics Department, Yeditepe University. Istanbul, Turkey.

https://doi.org/10.31871/WJRR.5.4.9 precisely how the future evolves out of the present. The law is absolute and, once present conditions are specified, there is no freedom or uncertainty in how the future will evolve," [2a]. The effectiveness and the limitations of Newtonian paradigm are described concisely by the passage:" The success of scientific theories from Newton through the present day is based on their use of a particular framework of explanation invented by Newton. This framework views nature as consisting of nothing but particles with timeless properties, whose motions and interactions are determined by timeless laws. The properties of the particles never change, and neither do the laws that act on them," [2b].

The brightest example of this style of thinking gave Pierre-Simon Laplace, who claimed that if he were given the precise positions and motions of all the atoms together with a description of forces acting on them, he could predict the future of the universe with total accuracy. Even more rigorous was Ludwig Boltzmann, who claimed," For the universe, the two directions of time are indistinguishable, just as in space there is no up and down," [2c].

In Modern physics the illustration of this approach can be found in the University text books on physics of the prominent American physicist R. Feynman [3]. For the proper understanding of the concept of time, which will be presented below, and for the realization of the importance of the advances that became feasible as a result of contribution of the outstanding personalities such as Ch. Darwin, A. R. Wallace, E. Mach, N. Bohr, K. Popper, A. Einstein, W. Pauli, some quotations from the Feynman text book will be presented below for comparison.

As the concepts of time and space, their symmetries and inter-relations are studied in Natural sciences since the time of Galileo Galilee, R. Feynman had to express his ideas on the subject explicitly and in a simple, transparent language in his course of lectures. In chapter 52 "Symmetry in Physical Laws" in [3a], Feynman writes:" The first thing we might try to do, for example, is to translate the phenomenon in space. If we do an experiment in a certain region, and then build another apparatus at another place in space then, whatever went on in one apparatus, in a certain order of time, will occur in the same way if we have arranged the same condition, with all due attention to the restrictions that we mentioned before: that all of those features of the environment which make it not behave the same way have also been moved over

In the same way, we also believe today that displacement in time will have no effect on physical laws. That means that if we build a certain apparatus and start it at a certain time, and then build the same apparatus and start it, say, three days later in the same condition, the two apparatus will go through the same motions in exactly the same way as a function of 
time no matter what the starting time, provided again, of course, that the relevant features of the environment are also modified appropriately in time."

And on the next page in [3a], we can find new assertions: "On a more advanced level we had another symmetry - the symmetry under uniform velocity in a straight line. That is to say - a rather remarkable effect - that if we have a piece of apparatus working a certain way and then take the same apparatus and put it in a car, and move the whole car, plus all the relevant surroundings, at a uniform velocity in a straight line, then so far as the phenomena inside the car are concerned there is no difference: all the laws of physics appear the same. We even know how to express this more technically, and that is that the mathematical equations of the physical laws must be unchanged under a Lorentz transformation.

Now the above-mentioned symmetries have all been of a geometrical nature, time and space being more or less the same, but there are other symmetries of a different kind. For example there is a symmetry which describes the fact that we can replace one atom by another of the same kind. The fact is that there are many, many atoms of the same type. Thus it does mean something to say that it makes no difference if we replace one atom by another of the same type. The so-called elementary particles of which the atoms are made are also identical particles in the above sense - all electrons are the same; all the protons are the same; all positive pions are the same; and so on."

And section 52-2 "Symmetry of space and time" Feynman concludes with the maintenance that,"if we look at the individual atoms themselves, the laws look completely reversible. This, of course, a much harder discovery to have made, but apparently it is true that the fundamental physical laws, on a microscopic and fundamental level, are completely reversible in time!"

In the next section 52-3 "Symmetry and conservation laws", when introducing the principles of quantum mechanics, Feynman writes (p.52-4): "The fact, for example, that the laws are symmetrical for translation in space when we add the principles of quantum mechanics, turns out to mean that momentum is conserved

That the laws are symmetrical under translation in time means, in quantum mechanics, that the energy is conserved.

Invariance under rotation through a fixed angle in space corresponds to the conservation of angular momentum.

These connections are very interesting and beautiful things, among the most beautiful and profound things in physics."

And this presentation he continues in [3b], chapter 17 (p.1): "In classical physics there are a number of quantities which are conserved - such a momentum, energy, and angular momentum. Conservation theorems about corresponding quantities also exist in quantum mechanics. The most beautiful thing of quantum mechanics is that the conservation theorems can, in a sense, be derived from something else, whereas in classical mechanics they are practically the starting points of the laws. In quantum mechanics, however, the conservation laws are very deeply related to the principle of superposition of amplitudes and to the symmetry of physical systems under various changes... The essential point is that the theorems about the conservation of all kinds of quantities are - in quantum mechanics - related to the symmetries of the system."

So, in accordance with the Feynman's textbook, "That the laws are symmetrical under translation in time means, in quantum mechanics, that the energy is conserved," and "that the laws are symmetrical for translation in space... turns out to mean that momentum is conserved." Thus, the conservations of energy and momentum form the fundamentals of quantum mechanics. But the theory of quantum mechanics, in general, and Feynman's textbook look incompatible with the observation of Paul Dirac, which was stating:" At the beginning of time the laws of Nature were probably very different from what they are now. Thus, we should consider the laws of Nature as continually changing with epoch, instead of as holding uniformly throughout space-time," [3d].

René Descartes (to whom the mankind is obliged for the invention of the "Descartes reference system" and for whom the philosophy was a thinking system that embodied all knowledge) presented all the knowledge in the form of a tree; of which Metaphysics is the root, Physics - the trunk, and all the other sciences - the branches that grow out of this trunk. If we use this scheme to compare the roots with the trunk, which is represented by the theoretical physics mainstream, we shall see that the trunk is crippled and deformed relative to the roots. Indeed, in G. W. Leibniz's Metaphysics, every material object, or "every simple Substance is by its nature a concentration and a living mirror of the whole Universe, according to its Point of view," [4a]. And the relational character of coexistence of material objects in the Universe, Gottfried Wilhelm Leibniz called in paragraph 59 of his "Monadology" that "universal harmony, according to which every substance exactly expresses all others through the relations it has with them," [5]. And in accordance with the ancient Greek dialectics of Heraclites of Ephesus that everything is changing, everything is in the state of flow, Leibniz in [5] (\$10) writes: "I assume also as admitted that every created being, and consequently the created simple substance (Monad), is subject to change, and further that this change is continuous in each." Meanwhile, in accordance with [2b], the theories based on Newtonian paradigm "view nature as consisting of nothing but particles with timeless properties, whose motions and interactions are determined by timeless laws." In these type of theories it is considered [3a] that "all electrons are the same; all the protons are the same; all positive pions are the same; and so on."

When defining the properties of the relational World, Leibniz writes in paragraphs 53-56 in [5] that "this interconnection, or this adapting of all created things to each one, and of each one to all the others, brings it about that each simple substance has relational properties that express all the others." And Leibniz maintains that the "right to claim existence being proportional to the perfection it contains," and this "is the reason for the existence of the best." Here, it should be taken into account that according to Leibniz, "a thing's perfection is simply the total amount of positive reality it contains," [5] (\$41). By these quotes, Leibniz was actually predicting the existence of the Natural evolution process, which was discovered experimentally by $\mathrm{Ch}$. Darwin and A. R. Wallace more than 140 years later, in 1858. In this respect, L. Smolin writes on p. XVI in [2]: "Darwinian evolutionary biology is the prototype for thinking in time, 
because at its heart is the realization that natural processes, developing in time, can lead to the creation of genuinely novel structures." Meanwhile, in theoretical physics, the motion of the three bodies, which are interacting gravitationally and which are not changing their properties in these interactions, cannot be obtained in the general case $(\mathrm{H}$. Poincaré and H. Bruns, 1887). So, the description of the evolution processes in Nature, where the properties of the interacting bodies are changing and their number is enormous, is clearly far beyond the possibilities of theoretical physics. Besides that, in the theories, which are based on Newtonian paradigm, only isolated systems are under investigation.

Describing this approximation, Smolin in [2] (p. 104) writes: "This division of the world into a dynamical and a static part is a fiction. The second part, assumed to be static, in reality consists of other dynamical entities outside the system being analyzed. By ignoring their dynamics and evolution, we create a framework within which we discover simple laws.

For most theories except general relativity, the fixed background includes the geometry of space and time. It also includes the choice of laws, as these are assumed to be changeless. Even general relativity, which describes a dynamical geometry, assumes other fixed structures, such as the topology and dimension of space."

The striking difference between Metaphysics and theoretical physics is in the role of mathematics. According to Leibniz’s "Monadology" (§§ 31-35): “There are two kinds of truths: those of reasoning and those of fact.

Truths of reasoning are necessary, and their opposite is impossible.

Truths of fact are contingent, and their opposite is possible.

When a truth of reasoning is necessary, the reason for it can be found by analysis. In Mathematics speculative Theorems and practical Canons are reduced by analysis to Definitions, Axioms and Postulates," where axioms and postulates are basic principles, which can't be proved within the structure of the theorem.

"What mathematicians do is to find sufficient reasons for the truth of mathematical propositions. "

And $\S \S 36-40$ in [5] we find:“ But a sufficient reason must also be found for contingent truths, truths of fact-for the series of things, spread across the universe of created things. The train of detailed facts about contingencies doesn't contain the sufficient reason for any contingent fact. For that we must look outside the sequence of contingencies. The sufficient or final reason must be outside of the sequence or series of particular contingent things, however infinite this series may be.

The final reason of things must be in a necessary substance, in which the variety of particular changes exists only eminently, as in its source; and this substance is a sufficient reason of all this variety of particulars, which are also connected together throughout. We may also hold that this supreme substance, which is unique, universal and necessary, nothing outside of it being independent of it - this substance, which is a pure sequence of possible being, must be illimitable and must contain as much reality as is possible."
For the material relational World, this supreme substance, which is unique, universal, and there is nothing outside of it being independent of it, and which is illimitable, and which contains as much reality as is possible, nowadays is called Universe (in agreement with [5], §§ 54-58).

So, if we compare the assertions in [5] of $\S \S 31-35$ with the ones of $\S \S 36-40$, we arrive to the conclusions of the famous K. Gödel's First incompleteness theorem [6], by which he demonstrated that the claims of the theories based on Newtonian paradigm possess no generality and thus cannot form the basis of Natural philosophy. So, the Feynman's discourses in his text book about the laws of physics, which are representing the mathematical relations of partial, incomplete theories [7], are the manifestations of the fact that at that time Feynman was not quite free from the idle metaphysical conceptions of Sir Isaac Newton expressed explicitly in [1] (in accordance with the terminology of E. Mach on page 224 in [8]).

The strenuous efforts of G. W. Leibniz, E. Mach, A. Einstein, W. Pauli and others to make free theoretical physics from the remnants of medieval philosophy were gradually giving results. For example, R. Feynman, only one year after publication of his text book, issued a printed a statement that was quite shocking for his colleagues: "I think I can safely say that nobody today understands quantum mechanics," [9]. It had much more serious impact among physicists than the earlier observation of A. Einstein, "The more success the quantum mechanics has, the sillier it looks," (Letter H. Zangger on May 20, 1912). Somewhat later, R. Feynman, when changing drastically his former views, was musing at one of his press-conferences: "The only field which has not admitted any evolutionary question is physics. Here are the laws, we say...but how did they get that way, in time? So, it might turn out that they are not same all the time and that there is a historical, evolutionary question," [2] (p. XXVI). Now, the views of Feynman (expressed in the last part of this quotation) are close to the position of the American philosopher Charles Sanders Peirce, who wrote in 1891: “To suppose universal laws of nature having no reason for their special forms, but standing inexplicable and irrational, is hardly a justifiable position. Uniformities are precisely the sort of facts that need to be accounted for. . . Law is par excellence the thing that wants a reason.

Now the only possible way of accounting for the laws of nature and for uniformity in general is to suppose them results of evolution," [10].. These views of Peirce clearly present a further development of the Leibniz's ideas about the relational material World.

And the first part of the last Feynman quotation is in close agreement with the preceding historical statement of A. Einstein: "No amount of experimentation can ever prove me right; a single experiment can prove me wrong". The real, tragic meaning of this statement follows from the published documents, in which Einstein was speaking about his "painful but inevitable resignation", when he realized that in his theories he was not able to overcome the Newtonian paradigm, and when according to R. Carnap, " Einstein thought that these scientific descriptions cannot possibly satisfy our human needs, " [11]. The importance of the last quote becomes obvious from the last official publication of A. Einstein [12], where he wrote: "The correctness of the theory is judged by the degree of agreement between the 
conclusions of the theory and human experience." The corresponding feature of the General Relativity Theory (GRT), Smolin in [2] (p. 71) describes in the following way: GRT "fits neatly into Newtonian paradigm. There is a space of possible configurations of the geometry and the matter together. Given the initial conditions, Einstein's equations determine the whole future geometry of a particular space-time and everything it contains.

And in general relativity, the whole history of the world is still represented by a mathematical object. The space-time of general relativity corresponds to a mathematical object much more complex than the three-dimensional Euclidean space of Newtonian theory. But seen as a block universe, it is timeless and pristine, with no distinction of future from past and no role for our awareness of the present." And Smolin concludes: "By removing the need for a clock outside the system, general relativity goes some distance toward a relational theory of physics. But still it is based on the Newtonian paradigm, as it can be formulated in terms of timeless laws acting on a timeless configuration space," [2] (p. 72).

By the form of his statement, "No amount of experimentation can ever prove me right..." A. Einstein wanted, probably, to demonstrate that he follows the philosophy of "critical rationalism" of K. Popper, the philosopher of science. In his philosophy, K. Popper maintains that no number of positive outcomes at the level of experimental testing can confirm a scientific theory, but a single experimental counterexample is decisive; it shows that the implication, which is derived from the particular theory, is false. In this case the theory is called falsified. Popper argues that a theory should be considered scientific if, and only if, it is falsifiable. According to Popper, the advance of scientific knowledge is an evolutionary process, in which the process of error elimination is similar to the natural selection process in biological evolution. So, any kind of rigorous experimental testing does not protect a scientific theory from the falsification in future, similar to the fact that the present day accommodation of biological species to the external conditions does not ensure their continued future survival. Especially, when taking into account Popper's position that scientific theories are abstract in nature and can be tested only indirectly, by the reference to their implications.

One of the fundamental A. Einstein's contributions into the development of physics is the introduction of a new (Popper - Einstein) criterion, which, on one hand, defines rigorously the relations between the experiment and the theory, clearly emphasizing the asymmetry in their positions: only experiment can refute a theory, but no theory is able to refute the other theory or to refute any experiment. On the other hand, this criterion gives an immense boost to the advance of physics, as it helps to use more effectively the material and human resources: only one experiment is sufficient to falsify the theory, and no more types of experiments are needed for that; no repairing activity can save a falsified theory, it should be substituted by a new theory, which is based on new approaches. But if a falsified theory has still some important practical applications, then new types of experiments are needed to reduce gradually the uncertainty in the range of the conditions, under which this "partial" theory can be applied within the specified spread of predicted uncertainties. The other part of the Einstein's immense contribution to physics was his own unprecedented example of falsification of all of his theories, just because "these scientific descriptions cannot possibly satisfy our human needs," or because the conclusions of his theories were not "in agreement with human experience." It should be clear that this criterion was the result of the support of the relational World, as according to Leibniz, for a contingent fact (or for a particular observation in the contemporary language) "the sufficient or final reason must be outside of the sequence or series of particular contingent things, however infinite this series may be."

"Thus the final reason of things must be in a necessary substance, in which the variety of particular changes exists only eminently, as in its source."

"Now this connexion or adaptation of all created things to each and of each to all, means that each simple substance has relations which express all the others, and, consequently, that it is a perpetual living mirror of the Universe," [5] (\$§ 37, 38 and 56).

The majority of the recently published books in physics are in support of the relational approach. For example, B. Greene writes: “The concept of symmetry's breaking, and its realization through the electroweak Higgs field, clearly plays a central role in particle physics and cosmology. Like the ether, a condensed Higgs field permeates space, sweeps through everything material, and as a non-removable feature of empty space, it redefines our conception of nothigness," [13]. Much further advances L Smolin in [2] (p. 215): "All the symmetries, so far posited by physicists, have turned out to be approximate or broken. Our universe is one where every moment of time, and every place at every moment, is uniquely distinguishable from any other. No moment ever repeats. Looked at in enough detail, every event in the universe is unique. In such a universe, there is never a complete realization of the conditions needed to make sense of the Newtonian paradigm."

But as no theory can refute the other theory, special attention has to be devoted to experimental studies, which are capable of the falsification of the existing theories. In this paper, the experimental studies of the thermal evolution process, which are far beyond the scope of description of the existing theories (as it is demonstrated here in "Conclusions and discussions"), are presented in the second section, and the consequences of these experimental results on existing theories are discussed in detail in the third section. The main features of the studies on the thermal evolution process are: the violations of symmetries in space and in time, which are observed in the experiments with purely electromagnetic (EM) types of the external influence on the system and with the EM detection of the result of this perturbation; the corresponding braking of the conservation laws of momentum and energy; the demonstration of the invalidity of the superposition principle for electromagnetic fields interacting with the material object; the demonstration of the existence of the thermal surface energy (TSE) and of the thermal hysteresis effect with well-defined hysteresis loops; the experimental proof that the thermal evolution process is the result of interactions of a huge number of material objects, which are constantly changing their properties in the process of interactions, with the inevitable consequence that the process cannot be described in principle in terms of mathematics. The paper also describes numerous types of 
experiments that falsify the existing theories based on the Newtonian paradigm and gives clear experimental support of the relational material World of G. W. Leibniz. The concept of time is formulated for the relational World, which is in agreement with the views of G. W. Leibniz, Ernst Mach and Daniel Kleppner and which is in agreement with the concepts of time existing nowadays in the other Natural sciences, such as Biology, Zoology, Geology and Astronomy.

\section{EXPERIMENT}

All these experiments were based on the original method of synchronous differential thermal measurements [14, 15], which was based on the periodic modulation of the input flux of energy to the investigated system and on the signal averaging procedure within one modulation cycle [16]. Using this method, the first observations and the studies of the thermal surface energy [16,17] and of the thermal evolution process were realized [18-21]. An experimental set-up of the present study, which is a natural continuation of the indicated investigations, is shown in Fig.1.

A $100-\mathrm{mm}$ steel gauge block (GB), supported by three polished spheres in the horizontal position, was located at the bottom of a closed Dewar that was in a temperature controlled, dark laboratory. The typical standard deviation of air temperature in the laboratory was $30-50 \mathrm{mK}$ on a daily basis, and the long-term temperature stability of the studied GB was determined by the temperature stabilization system of the lab. The large time constant of the system in the Dewar reduced only the rate of the temperature variations, so that for precise measurements, the magnitudes of the temperature velocities were considered to be acceptable if they were within $80 \mu \mathrm{K}$ per minute.

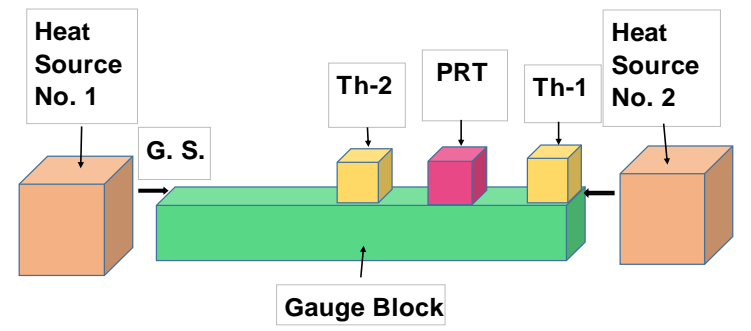

Fig.1. Block-diagram of the experimental system located inside a Dewar: steel gauge block, with two gauging surfaces (G.S); platinum resistance thermometer (PRT) in copper adapter; thermistors Th- 1 and Th-2 in copper adapters; auxiliary heat sources No.1 and No.2 are used to produce a systematic temperature bias between the positions of thermistors No.1 and No. 2 and are installed at some distance from the gauging surfaces. (See text for other details).

On the larger side surface of the GB, with the dimensions $35 \times 100 \mathrm{~mm}$ and the height of $9 \mathrm{~mm}$, three thermometers were installed. Each thermometer was located inside a copper adapter covering the whole width of the block, so that it measured a mean value of the surface temperature along the width of the block. The centre position of the measurement system on the GB surface (Fig.1) was occupied by the adapter of a $100 \mathrm{Ohm}$ platinum resistance thermometer (PRT). Symmetrically relative to it, the adapters of $18 \mathrm{kOhm}$ thermistors (with negative temperature coefficients) were located. The output signals of these thermistors (belonging to channels 1 and 2) were used in the synchronous differential thermal measurements. The separations between the corresponding sides of the adapters were $13.5 \mathrm{~mm}$, and one of the side surfaces of the adapter of the thermistor of Ch-1 (Th-1) was located in the plane of the gauging surface of the steel GB (as shown in Fig.1). For the widths of all adapters equal to $9 \mathrm{~mm}$ and the symmetric locations of thermometers in adapters, these experimental conditions corresponded to the separation of the axis of thermistor- 1 equal to $4.5 \mathrm{~mm}$ from the nearest gauging surface. The separation of the axis of the sensor Th2 from the same gauging surface was $49.5 \mathrm{~mm}$, so that this sensor was located very close to the centre of the block surface.

Both thermistors were connected to high-precision Hp-3458A multi-meters, and the measured values of the resistances of the thermistors as functions of time were stored in computer. The key feature of these temperature measurements was the special procedure of calibration of thermometers. Each thermistor (sealed in a copper adapter) was calibrated together with the particular unit of the multi-meter on the surface of a long steel gauge block that was located inside the temperature stabilized Kösters interferometer $[14,15]$. As a reference in these calibrations we used a 25-Ohm Rosemount standard platinum resistance thermometer (SPRT 162E, produced in USA) and an automatic precision DC MI-bridge T615 (Canada), or the Guildline current comparator 9975 (when necessary). The calibration procedure was described in some detail in [14, 22, 23] and was giving the opportunity to measure the temperature of the surface of a steel artifact in the close proximity to the boundary of the thermistor adapter. In the calibration procedure realized in these experiments, the measured resistance value of a thermistor, corresponding to each temperature of the gauge block surface, was compared with the resistance of a temperature stabilized standard resistor with the nominal value of $10-\mathrm{kOhm}$. Thus, the ratio of the resistance value of the particular thermistor to the resistance of the standard resistor was used in the calibration equation as a function of surface temperature. When using Hp-3458A multi-meters, for the half of a measurement time interval, the measurement current of the instrument was equal to $50 \mu \mathrm{A}$; during the other half, when the current was equal to zero, the electronic offset of the instrument was precisely measured, and in this way, the measurement result was compensated for the instrument's offset. It is clear that the effective self-heating effect of the thermistor in this scheme is only one half of the heating effect of the thermometer, which corresponds to the case of the use of a bridge with current reversal of the same magnitude. Besides that, due to the fast response of the multi-meters relative to the bridges, precise time synchronization between the measurements in thermistor channels was appropriately realized, which contributed significantly to the accuracy improvement of our differential thermal measurements.

As a source of the energy flux modulation, in our measurements we used a periodic current variation in a 100-Ohm platinum resistance thermometer (PRT). The PRT was connected to the MI-bridge, which realized the precise current modulation in accordance with our program. In this experiment, the measurement current was periodically changed from $1 \mathrm{~mA}$ to $5 \mathrm{~mA}$. The print-screen of the program, which was developed specially for realization of 


\section{Experimental Demonstration of the Relational Character of Time in Natural Processes; Concept of Time in the Relational World}

measurements, for storing, and for processing of the results of the measurements, is presented in Fig.2. The duration of the presented record is 200 minutes. So, more than three 60-minute modulation cycles can be observed on it. The duration of the heating period of the modulation cycle (corresponding to $\mathrm{I}=5 \mathrm{~mA}$ ) was 15 minute, and the cooling period (at $\mathrm{I}=1 \mathrm{~mA}$ ) was three times longer. In Fig.2, the record of a signal with the faster time response corresponds to the PRT channel. The two other records correspond to the resistance measurements of the two thermistors, which have the negative thermal resistance coefficients. The increase of the temperatures in the records corresponds to the higher part of the print-screen for the PRT and to the lower part of the screen for the thermistors.

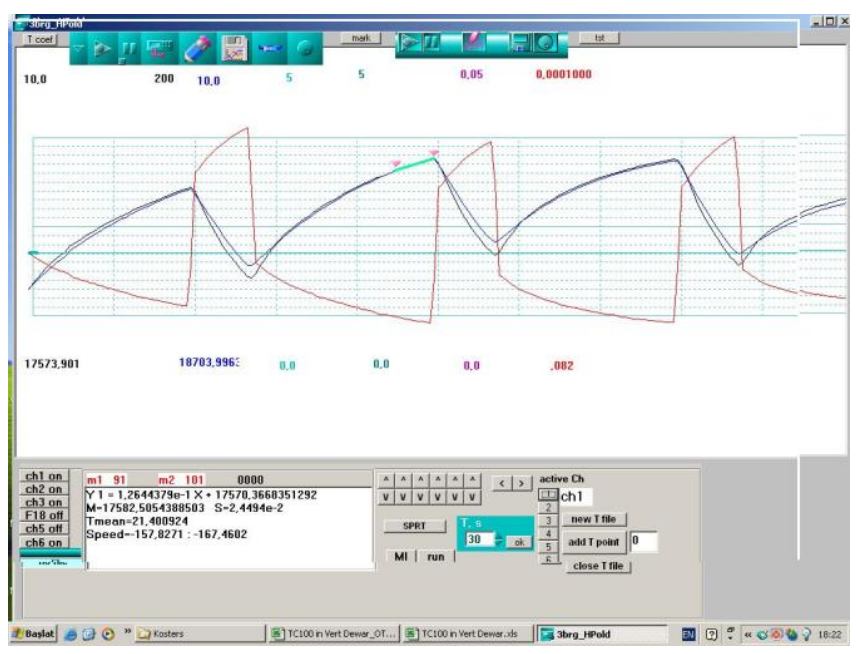

Fig.2. Simultaneous records of the resistance variations of a platinum resistance thermometer (PRT) and of two thermistors Th- 1 and Th-2, which are located symmetrically relative to the PRT on the surface of the gauge block. During the current modulation cycle in the PRT, its current for $1 / 4$ of the modulation period is kept at the level of $5 \mathrm{~mA}$ and $3 / 4$ of the period is kept at $1 \mathrm{~mA}$. The sensitivities of the thermistors are, practically, equal. Two cursors (shown by triangles) define the averaging time for the mean values of temperature quantities. (See text for other details).

Two cursors of the program, shown in Fig.2 as triangles, were used to specify the desired time interval for averaging the results of measurements. In accordance with the calibration equation, the program was calculating the mean values of the temperature and of the thermal velocity in each channel for the selected time interval. The results of the calculations for each channel were presented in the program's window. After converting the resistance curves as functions of time into the corresponding temperature dependences versus time, the obtained plots give us important information about the temperature evolution processes, which occurred in the steel block in vicinities of the sensors Th1 and Th2. But here, it is worth noting that the most important, fascinating result of this type of studies, i.e., braking of symmetries in time and in space in thermal processes [18, 19], which unambiguously falsify the Newtonian paradigm, can be picked up directly from the unprocessed experimental data, i.e., the records of Fig.2. In this case, even the conversion of the resistance variations of the thermistors into the corresponding temperature variations are not needed for a while: the differences in the sensitivities of the thermistors are quite small and the results of this conversion can not be quite clearly discerned in the scale of Fig.2. The only prerequisites for the observation of the effect are the use of synchronous differential thermal measurements and the realization of the square-wave modulation of the fluxes of energy and momentum, which are delivered to the gauge block by EM field.

First, from the thermistor records of Fig. 2 it follows that as the slopes of the curves at the end of the cooling cycles are practically equal, so we can conclude that the fluxes of energy, which are radiated per unit time into the outer space by the block surfaces in the vicinities of the thermistors 1 and 2 , are also practically equal. (Indeed, these fluxes of energy, when absorbed by thermistors and converted into thermometer resistance, result in the same values of the rates of the resistance variations). But the situation is absolutely different during the heating period of the cycle: in this case, the slope of the record of thermistor- 1 is clearly larger than that of thermistor-2, and consequently, the corresponding part of the gauge block surface emits larger flux of energy. And this occurs is in spite of the fact that the distances of the thermistors from the modulation source are exactly the same. (The separations between of the adapter of the PRT and the adapters of thermistors were established with the help of a short gauge block with an appropriate nominal length.) This means that the symmetry in space of the properties of the initially homogeneous material is lost when the gauge block absorbs the fluxes of the energy and momentum of the external EM field [16]. At the same time, when the photons of EM field are absorbed in the material artifact, in accordance with [24] inside the artifact do appear the fluxes of energy and momentum of the field-particle system, which can be detected by their thermal radiation by two thermometers located inside the material artifact along the direction of the energy propagation [23]. And as the velocities of the energy and momentum propagation are much smaller than the velocity of the EM field propagation in a free space [24, 20], the corresponding deficit of the linear momentum is transferred from the gauge block to the Dewar, further to the environment, and finally to the Earth through the block suspension device [19]. Thus, it follows from the studies of the thermal evolution process that when there is no symmetry in space, there is no conservation of linear momentum of the system, and vice versa.

Here, it should be mentioned that the violation of spatial symmetry is the result of the appearance of the thermal surface energy [16], which arises in the vicinity of the boundary of the artifact, when the excessive fluxes of energy and momentum of the external EM field (relative to the thermal equilibrium conditions) are absorbed in the artifact For the experiments, presented here and in $[16,17]$, the main reason for the arising surface energy is the reflection of the momentum of the field-particle system from the gauging surface of the block [19]. This type of thermal surface energy is characterized by well-defined hysteresis loops [17-19]. And the hysteresis loop means simultaneously that, one hand, the process is irreversible in time, and on the other hand, the energy of the system is not conserved [19]. The hysteresis curves corresponding to the records of Fig.2, which will be presented below, and they will give another confirmation that the irreversible time and the violation of "the law of conservation of energy of the system" are the properties of the 
thermal evolution process that are observed experimentally simultaneously. The main result of the presented studies is the experimental demonstration that an external, auxiliary source of broadband, thermal radiation modifies significantly the successive states of a thermal system, or in other words, changes the hysteresis effect at a particular point of a material artifact, thus inevitably modifying the dependence in time of a thermal evolution process. But now we shall show that the specific features of a particular hysteresis effect can be picked up directly from the records of Fig.2.

First, it follows from the presented plots that the response of the artifact to the square-wave modulation of the fluxes of energy and momentum is not linear and occurs with the evident delay in time. And nonlinearity and the time delay of the system are the typical for all types of hysteresis effect. Second, for the beginning of the cooling period, the magnitude of the slope of the curve for the thermistor- 1 is substantially larger than the magnitude of the slope of the same curve at the end of the heating period of the modulation cycle. It means that the cooling at the beginning of the cooling period is faster than the heating at the end of the heating period of the cycle. And this is again the characteristic property of a well-defined hysteresis loop [21]. Third, one can detect some delay in time (of about 1.5-2 minutes) that is required for the increase of the differential thermal signal. In accordance with $[19,21]$, this time is necessary for the wave-momentum (introduced by Loudon, Allen \& Nelson in [24]) to reach the thermistor- 1 after the reflection of the wave from the gauging surface of the block, thus realizing the main mechanism of creation of the hysteresis effect that is typical under the present experimental conditions. These properties will be demonstrated in detail on the experimental plots presented below.

At last, it is worth paying attention to the fact that during the cooling period of the modulation cycle the loss of energy is larger for the surface area in the vicinity of thermistor-1 than the corresponding loss in the vicinity of thermistor- 2 . It means that during the cooling period of the cycle, the thermal energy is also continuously removed from the gauge block by the air-conditioning system, so that the studied periodic process, which is characterized by the loss of energy of the oriented motion of the field-particle system in each cycle, is irreversible in time in agreement with the laws of thermodynamics [17]. Indeed, no equilibrium thermal system (staying at any temperature) can result spontaneously in a cyclic, oriented motion (for example, lifting in a cyclic way some weight, as this process is strictly forbidden in accordance with the Plank's formulation of the Second law of thermodynamics). Thus, the results of our experiments (including those presented below) give a clear indication that the real processes, which are characterized by some losses of fluxes of EM energy and momentum, are irreversible in time and have no spatial symmetry.

As pointed out above, very accurate quantitative results can be obtained after the conversions of the resistance records of Fig. 2 into the corresponding temperature dependences as functions of time. For example, for the last measured point in the cooling period of the second cycle of Fig.2, the mean temperatures in channels 1 and 2 were found to be equal to $20.774135^{\circ} \mathrm{C}$ and $20.777013{ }^{\circ} \mathrm{C}$, respectively (for the 5 minutes averaging time). The difference in temperatures
$\mathbf{T}[\mathbf{1 , 2}]$ was about $-2878 \mu \mathrm{K}$. After the end of the heating period, the measured values in the channels 1 and 2 were $20.78392^{\circ} \mathrm{C}$ and $20.78464^{\circ} \mathrm{C}$, corresponding to the value of $\mathbf{T}[\mathbf{1 , 2}]=-715 \mu \mathrm{K}$. So, the induced temperature variation, caused by the increase of the modulation current in the PRT and denoted here by the symbol $\Delta \mathbf{T}[\mathbf{1 , 2}]$, in this case is approximately equal to $2200 \mu \mathrm{K}$. In accordance with [16, 17], the quantity $\Delta \mathbf{T}[\mathbf{1 , 2}]$ can be used to describe the process of the build-up of the thermal surface energy (TSE), which arises close to the boundary of the artifact during the heating period of the modulation cycle, when the fluxes of energy and momentum of the EM field (generated in the PRT) are absorbed in the gauge block. The quantity $\Delta \mathbf{T}[\mathbf{1 , 2}]$ is a vector, which carries information about the relative positions of the thermistors 1 and 2. The comparison of thermistor records in Fig.2 shows that the quantity $\mathbf{\Delta T}[\mathbf{1 , 2}]$ is positive during the heating period of the modulation cycle (at $\mathrm{I}=5 \mathrm{~mA}$ ). It means that the average flux of energy during this time period is larger to the elementary volumes in the vicinity of the sensor Th-1, than the corresponding energy flux to the volumes in the vicinity of Th-2. Thus, it is confirmed quantitatively that the present experimental conditions, the spatial symmetry in a homogeneous material artifact is lost when this artifact is exposed to external fluxes of energy and momentum of EM field, in agreement with [16, 17]. The synchronous differential thermal measurements also show that at the beginning of the cooling period of the modulation cycle (at $\mathrm{I}=1 \mathrm{~mA}$ ), the quantity $\Delta \mathbf{T}[\mathbf{1 , 2}]$ is becoming negative, so that there is already an excessive cooling of the elementary volumes in the vicinity of Th- 1 relative to the corresponding temperature variations of the elementary volumes in the vicinity of Th-2. By the end of the cooling period the homogeneity of the spatial properties of the artifact is, practically, restored.

After converting the three resistance records of Fig. 2 into the temperature dependences versus time, we obtain three records of the irreversible in time evolution processes, specific for three areas of the artifact surface during several modulation cycles. And at each observation point, the evolution process can be represented by the individual curve, which describes the successive states of the system evolving in time (in response to the rectangular type of the modulation signal used in our experiments). And it is important to emphasize that each cycle of the individual curve is always somewhat different from the others, just because the process is irreversible in time [19]. Naturally, the cycles, which are corresponding to different thermistor channels, are different from each other. First, this difference arises due to the manifestation of the surface energy, whose magnitude depends critically on the distance from the gauging surface [18]. Second, there is always a contribution of inevitable external thermal perturbations, whose result varies with the variation of the point of observation. The latter effect can be illustrated by the differential thermal measurements, when comparing the data-points for two channels, which correspond to the last ten minutes of the cooling periods of the adjacent cycles. (As it follows from [19], the contribution of the surface energy is quite negligible for this time interval.) For example, when comparing the last 10-minutes points for the cycles 1 and 2 in Fig.2, we find that the variations of the readings of thermistors 1 and 2 during 1 hour time interval were $-3773 \mu \mathrm{K}$ and $-3521 \mu \mathrm{K}$, respectively. The corresponding 


\section{Experimental Demonstration of the Relational Character of Time in Natural Processes; Concept of Time in the Relational World}

mean temperature velocities (for 1 hour averaging time) were $-62.9 \mu \mathrm{K} /$ minute (for channel 1 ) and $-58.7 \mu \mathrm{K} /$ minute (for the second channel). The system was cooling, and the temperature variations were larger in channel 1. The situation (characterized by the same set of parameters) was somewhat different for the cycles 2 and 3. During this time interval, the system was cooling much slower, and the temperature variations in channel 1 were smaller than in the other channel: $-15 \mu \mathrm{K}$ (channel 1) relative to $-137 \mu \mathrm{K}$ (channel 2); the mean temperature velocities were $-0.25 \mu \mathrm{K} /$ minute (channel 1) and $-2.28 \mu \mathrm{K} /$ minute (channel 2). At last, for the cycles 3 and 4 all the parts of the system were definitely heating, and the variations, which were recorded in channell, were again larger: $4223 \mu \mathrm{K}$ (channel 1) relative to $4158 \mu \mathrm{K}$ (channel 2); the corresponding thermal velocities for 1 hour measuring time were $70.4 \mu \mathrm{K} /$ minute and $69.3 \mu \mathrm{K} /$ minute, respectively.

These measurement results clearly demonstrate that the thermal evolution process is not only affected by the modulation signal, but it is influenced by numerous thermal perturbations, so that the process evolves both in time and in space. It is also known from [21, 20] that the form and the main parameters of the hysteresis loop, which is successfully used to characterize quantitatively the thermal evolution process, critically depend on the time interval after the application of the modulation signal, depend on the presence of additional sources of thermal radiation, and dramatically depend on the choice of the spatial positions of the thermometers on the artifact surface. It should be also emphasized that the thermal evolution process and the hysteresis effect cannot be adequately described by any mathematical formulae (obtained on the basis of some physical theory) just because the number of interacting material bodies in the evolution process is too big, and the properties of these bodies are continuously changing during the interaction process [19]. In this respect it should be reminded that in theoretical physics, even for the relatively simple case of three bodies, which obey the Newton's law of gravitation and whose properties are assumed to be without any change during the interaction process, the general solution cannot be found [8] (Ch.11).

But it is clear that for the constantly changing in time curve of the evolution process, which depends on the huge number of external parameters that cannot be measured accurately simultaneously, is not that useful for a detailed analysis. In order to reduce the effect of unpredictable thermal perturbations on the measurement results, the following procedures were applied [17].

First, we used synchronous temperature measurements at two locations, thus realizing the differential thermal measurements, which are indispensable for the studies of the energy propagation inside a material artifact and which reduce significantly the effect of temperature variations of the artifact as a whole.

Second, by using the square-wave input power modulation, only relatively fast temperature variations (generated by the modulation procedure) were practically detected, and the effect of the slowly changing thermal background was significantly suppressed.

Third, high-precision measurements of the vector quantity $\mathbf{\Delta} \mathbf{T}[\mathbf{1 , 2}]$ (which was used in [16] to characterize the thermal surface energy) were obtained by averaging of the measurement results over a large number of modulation cycles. In this case, a proper sequence of cycles for the analysis can be selected, when the same number of cycles corresponded to the time periods of slight heating of the system in the Dewar, and the same number of cycles corresponded to the periods of, approximately, the same level of cooling of the system. This type of sequence can be illustrated by the choice of the cycles 2, 3 and 4 in Fig. 2.

Fourth, to study of the effect of external sources of EM radiation on the thermal evolution process, generated by the modulation current in the PRT, we used modeling of the external thermal conditions. For this purpose, two equal, auxiliary resistors were installed inside the Dewar system, symmetrically relative to the gauging surfaces of the block (Fig.1). A stabilized current in one of them was used to produce the desired temperature difference $\mathbf{T}[\mathbf{1 , 2}]$ between the thermistors of channels 1 and 2. Under our experimental conditions, the temperature variations, which were produced by an auxiliary source, surpassed at least by an order of magnitude the temperature variations caused by uncontrolled external thermal perturbations. In this case, the obtained time dependence of the thermal evolution process was defined by the effect of an auxiliary energy source, and the effect of the thermal perturbations was relatively small due to the proper Dewar isolation and the averaging procedure over several modulation cycles. The following plots will demonstrate significant advantages of the differential thermal measurements, and some results of the analysis of the experimental data will be presented.

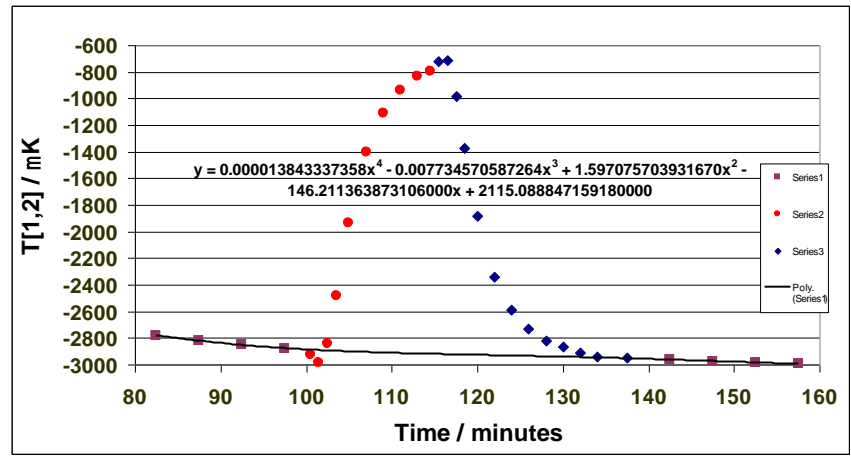

Fig.3. The dependence on time of the temperature difference $\mathbf{T}[1,2]$ between the thermistors Th- 1 and Th-2. The points, which are corresponding to the heating period, are presented by dots; the points of the cooling period of the modulation cycle are shown by rhombi and squares. The reference points, to which the polynomial fit is calculated, are shown as squares. The equation of the fit is presented in the inset. The solid line on the plot shows the reference function, relative to which the quantity $\Delta \mathbf{T}[\mathbf{1 , 2}]$ is measured. (See text for other details).

In Fig.3, the direct results of synchronous differential thermal measurements are presented that correspond to the third modulation cycle of Fig.2. In Fig.3, the difference in the recorded values of temperature $\mathbf{T}[\mathbf{1}, \mathbf{2}]$ in the channels 1 and 2 is presented as a function of time scale, which is generated by a crystal oscillator of our computer. The data-points (corresponding to the heating period of the modulation cycle at $\mathrm{I}=5 \mathrm{~mA}$ in the PRT) are shown by circles. The cooling period of the cycle (at $\mathrm{I}=1 \mathrm{~mA}$ ) is presented by rhombi and squares. The data-points, which correspond to the last 20 minutes of the cooling cycle, are called reference points and are shown as squares. As an example, the fourth order 
polynomial fit was calculated for all of the reference points of the modulation cycles 2 and 3 of Fig.2. The corresponding fit is shown in Fig.3 as a solid line (together with its equation). The calculated deviation of the synchronously measured temperature difference in the channels $\mathbf{T}[\mathbf{1 , 2}]$ (at some specified time) relative to the fit (at the same time) forms a new physical quantity, which is denoted by $\Delta \mathbf{T}[\mathbf{1 , 2}]$. This quantity $\Delta \mathbf{T}[\mathbf{1 , 2}]$ describes the differential temperature variations in the channels, which are induced by the current modulation in the PRT and which are practically free from the slow temperature perturbations in the system. The corresponding dependence of the quantity $\Delta \mathbf{T}[\mathbf{1 , 2}]$ as a function of time is presented in Fig.4a. Here, the standard deviation of the reference points relative to the fit, which characterizes the "noise" level in this experiment, is found to be $2.7 \mu \mathrm{K}$. Meanwhile, the maximum value of the quantity $\Delta \mathbf{T}[\mathbf{1 , 2}]$, which characterizes the level of the arising surface energy (generated by the energy flux from the PRT) is about $2207 \mu \mathrm{K}$. The uncertainty of the latter value is less than $10 \mu \mathrm{K}$.

As it also follows from the plot of Fig.4a, the maximum value of $\Delta \mathbf{T}[\mathbf{1 , 2}]$ is observed during the cooling period of the modulation cycle in the computer time scale, approximately 1.5 minutes after the decrease of the modulation current from $5 \mathrm{~mA}$ to $1 \mathrm{~mA}$ in the PRT. As shown experimentally in [20], the main reason for the arising thermal surface energy is the reflection of the oriented motion of the field-particle system (or the wave-momentum in terminology of [24]) from the gauging surface of the block. So, the indicated time delay characterizes the propagation time of the energy-momentum fluxes of the field-particle system from the PRT to the gauging surface and back to thermistor 1 . The maximum value of the surface energy $(2207 \mu \mathrm{K})$ corresponds to the experimental conditions when there was some temperature difference $\mathbf{T}[\mathbf{1}, \mathbf{2}]$ between the positions of the sensors Th-1 and Th-2. As it follows from Fig.3, at the beginning of the heating period there was the temperature difference $\mathbf{T}[\mathbf{1 , 2}]$ of about $-2.9 \mathrm{mK}$, which was created by an auxiliary heat source No.1 (switched on in advance). Thus, the experimental results of Fig.4a correspond to the case when the energy-momentum fluxes, generated by the increase of the modulation current in the PRT and reflected from the gauging surface, were propagating in the opposite direction to the previously existing energy flux, which was produced by the auxiliary heat source-1.

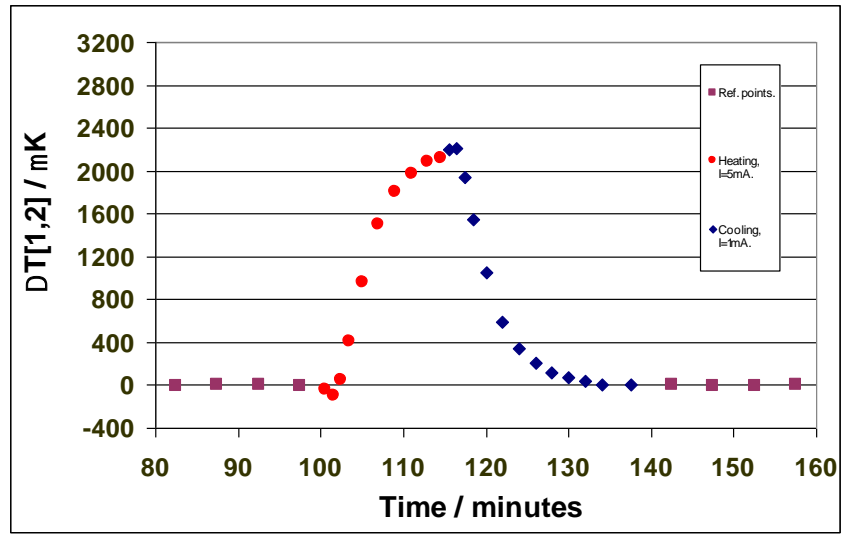

Fig.4a. The dependence of the quantity $\Delta \mathbf{T}[\mathbf{1 , 2}]$ (describing the thermal surface energy) as function of time is presented during the whole modulation cycle for the temperature difference $\mathbf{T}[\mathbf{1 , 2}]$ equal to $-2.9 \mathrm{mK}$.

To find the effect of an auxiliary heat source on the parameters of the thermal evolution process, the next experiments were performed with the opposite sign of the temperature difference $\mathbf{T}[\mathbf{1 , 2}]$ between the positions of thermistors 1 and 2. Now, the auxiliary energy source No.2, which was located closer to the gauging surface in the vicinity of the thermistor-2, was switched on. The current in the heat source was adjusted for the desired temperature difference $\mathbf{T}[\mathbf{1 , 2}]$. In this experiment, the dependence of the induced temperature difference $\Delta \mathbf{T}[\mathbf{1 , 2}]$ (Fig.4b) corresponds to the conditions when at the end of the cooling period of the modulation cycle the temperature difference $\mathbf{T}[\mathbf{1 , 2}]$ was $16.2 \mathrm{mK}$. So in this case, the temperature difference between the two locations on the block surface, induced by the current modulation in the PRT, was observed when inside the metallic block there was already an energy flux, which was propagating in the direction from the gauging surface into the bulk material as a result of the operation of the auxiliary energy source No.2. As it follows from the plot of Fig.4b, the magnitude of the surface energy has increased significantly in comparison with the corresponding result of Fig.4a.
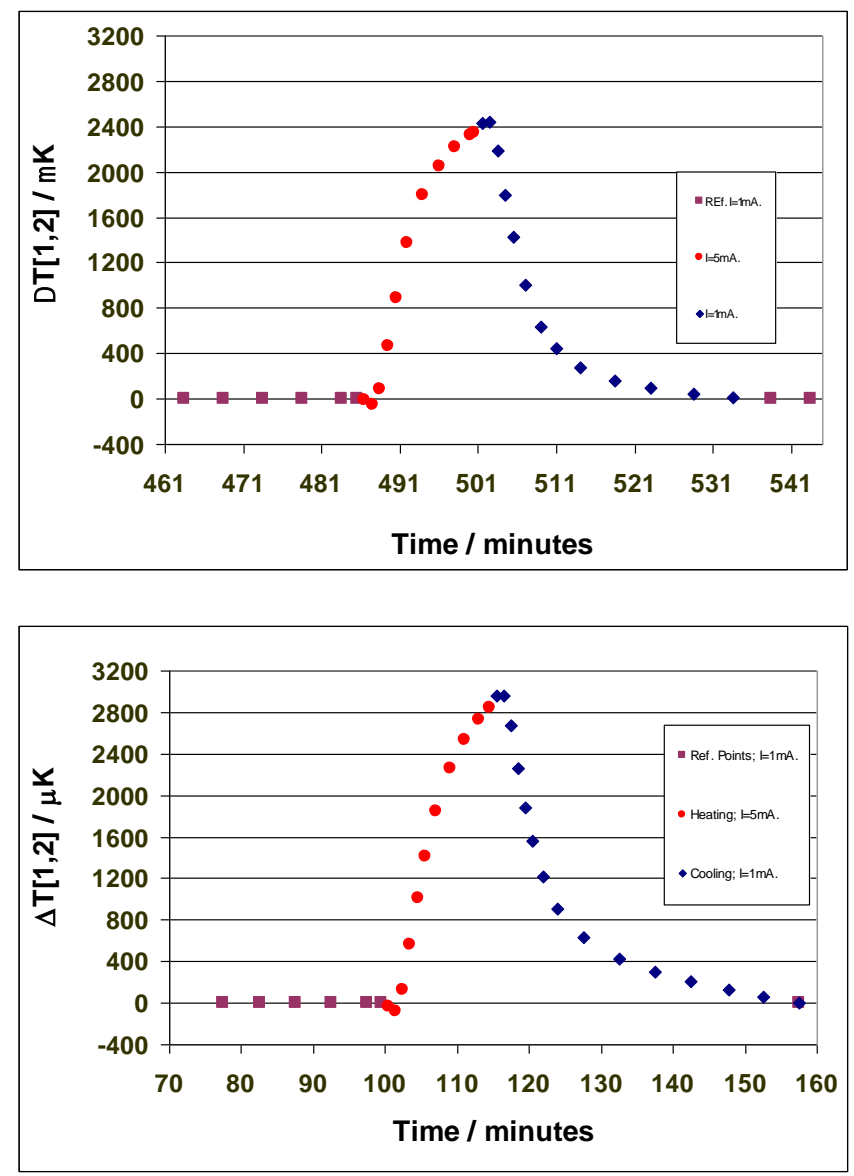

Figs.4b-4c. The dependences of the quantity $\Delta \mathbf{T}[\mathbf{1 , 2}]$ as functions of time are presented for the temperature differences $\mathbf{T}[\mathbf{1 , 2}]$ equal to $16.3 \mathrm{mK}$ and $57.1 \mathrm{mK}$, respectively.

Now the maximum value of the quantity $\Delta \mathbf{T}[\mathbf{1 , 2}]$, observed again at the time interval of 1.5 minutes after the decrease of the PRT current from $5 \mathrm{~mA}$ to $1 \mathrm{~mA}$, is about $2445 \mu \mathrm{K}$. The increase of the value relative to the first 


\section{Experimental Demonstration of the Relational Character of Time in Natural Processes; Concept of Time in the Relational World}

experiment is about $238 \mu \mathrm{K}$. The estimated uncertainty in the measurement of this difference is about $2-3 \mu \mathrm{K}$, only. But besides the change of the magnitude of the surface energy, from the comparison of the plots in Figs. $4 \mathrm{a}$ and $4 \mathrm{~b}$, one can detect clearly the fact (which is of primary importance for this study) that the sequence of states of the system, or the time dependence of the thermal evolution process is modified by an auxiliary energy source. For example, the area between the curve $\Delta \mathbf{T}[\mathbf{1}, 2]$ and the abscissa axis in Figs.4a-4b, which characterizes the excessive energy radiated by the system as a result of the arising thermal surface energy, is definitely increasing with the increase of the quantity $\mathbf{T}[\mathbf{1 , 2}]$. This means that the larger is the radiated power of the auxiliary source No.2, the shorter is the time interval, during which we are able to detect that this process is irreversible in time.

To illustrate the precision of the presented measurements and the validity of the results of the analysis of the experimental data, we performed the third experiment, when the temperature difference $\mathbf{T}[\mathbf{1 , 2}]$ was increased to $57.1 \mathrm{mK}$. The corresponding dependence of the induced temperature difference $\Delta \mathbf{T}[\mathbf{1}, \mathbf{2}]$ as function of time for the whole modulation cycle is shown in Fig.4c. It is obvious from the presented plot that the thermal surface energy has further increased relative to the previously performed experiments. The maximum value of the quantity $\Delta \mathbf{T}[\mathbf{1 , 2}]$, which is again observed at the beginning of the cooling period of the modulation cycle, is now reaching the value of $2956 \mu \mathrm{K}$. It means that with the increase of the temperature difference $\mathbf{T}[\mathbf{1 , 2}]$ from $-2.9 \mathrm{mk}$ to $57.1 \mathrm{mK}$ the surface energy has increased by approximately 34\%. And this is a clear experimental confirmation that the principle of superposition inside material objects is not valid for EM fields [16].

Comparison of the plots of the Figs. $4 \mathrm{a}-4 \mathrm{c}$ clearly shows that the form of the curves describing the thermal evolution process is changed as a result of an additional, external heat source. The analysis of the curves in Fig. 4 indicates that the slopes of the dependencies as functions of time, elapsed after the application of the modulation signal, are affected by the auxiliary energy sources. For example, the signal in Fig.4a during the heating period ( 15 minutes) is almost reaching the maximum value, which can be obtained for longer modulation cycles (see Fig.2 in [17]). It means that the excessive energy flux, which is responsible for the creation of thermal surface energy (TSE), diminishes almost to zero during this time interval (Fig.3 in [17]). Meanwhile here, in Fig.4b and especially in Fig.4c, this excessive energy flux is still present, and the magnitude of TSE could increase considerably for longer modulation cycles. We can introduce the parameter, which characterizes quantitatively the evolution process at the end of the heating period, defining the quantity $\delta \mathrm{T}$ as the difference between the maximum value of the quantity $\Delta \mathbf{T}[\mathbf{1}, \mathbf{2}]$ (obtained already during the cooling period) with the last value of $\Delta \mathbf{T}[\mathbf{1}, \mathbf{2}]$, which is observed during the heating period of the modulation cycle and which is obtained 0.5 minute before the decrease of the modulation current in the PRT. It follows from the Figs. $4 a-4 c$ that the parameter $\delta \mathrm{T}$ is steadily increasing with the increase of the temperature difference $\mathbf{T}[\mathbf{1 , 2}]$. The variations of $\mathbf{T}[\mathbf{1 , 2}]$ from $-2.9 \mathrm{mK}$ to $16.2 \mathrm{mK}$ and then to $57.1 \mathrm{mK}$ were accompanied by the increase of $\delta \mathrm{T}$ from $84 \mu \mathrm{K}$ to $100 \mu \mathrm{K}$ and then to $115 \mu \mathrm{K}$, respectively. The dependence of parameter $\delta \mathrm{T}$ as a function of temperature difference between the locations of the thermistors $\mathbf{T}[\mathbf{1 , 2}]$ is clearly nonlinear.

The interaction between the heat sources participating in the thermal evolution process is characterized quantitatively by the plots of Figs.5a and 5b. Also, the levels of uncertainties, which are typical for the measurements presented in Figs. $4 a-4 c$, are illustrated by these figures. In Fig.5a the surface thermal energy, which is characterized by the maximum value of the quantity $\Delta \mathbf{T}[\mathbf{1 , 2}]$ and which is observed during the whole modulation period, is presented as a function of the temperature difference $\mathbf{T}[\mathbf{1 , 2}]$ created by the auxiliary energy sources. As it follows from this plot, the principle of superposition for the external EM fields is not valid in case of the thermal evolution process, as the effect, which is generated by the modulation current in the PRT, is influenced by the direction and by the magnitude of the energy flux in the artifact that is created by the auxiliary energy source. The dependence $\Delta \mathbf{T}[\mathbf{1 , 2}]$ versus $\mathbf{T}[\mathbf{1 , 2}]$ is linear in the whole range of studied parameters. Our experiment covers both negative and positive values of $\mathbf{T}[\mathbf{1 , 2}]$ corresponding to different directions of the energy fluxes in the block. It should be noted here, that the total range of the variation of the surface energy in Fig.5a is about $750 \mu \mathrm{K}$, while the standard deviation of the measured points relative to the fit is $0.36 \mu \mathrm{K}$, only.
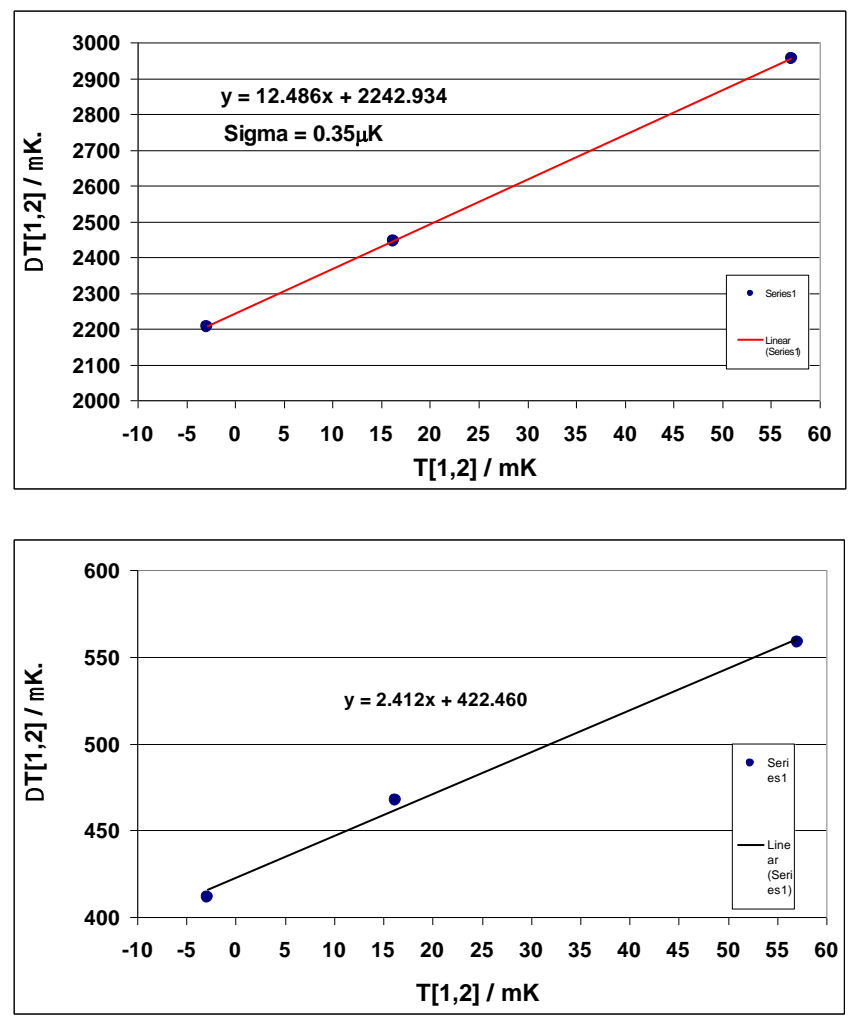

Figs.5a-5b. The dependences of the thermal surface energy $\Delta \mathbf{T}[\mathbf{1 , 2}]$ on the temperature difference between the locations of the thermistors $\mathbf{T}[\mathbf{1 , 2}]$ that are measured for the time intervals of 16.5 and 3.5 minutes after the increase of the modulation current in the PRT, respectively.

The slope of the plot characterizes the level of nonlinear interaction between the two sources of EM radiation as a result of the presence of the steel gauge block. When both variables in Fig.5a are measured in the same units, the slope becomes equal to $1.25 \%$, characterizing the nonlinear 
properties of the material object under the specified experimental conditions. And one of the fundamental results of the studies of the thermal surface energy is the demonstration that the properties of a material artifact and its response are continuously changing in time in the presence of the external EM fields [19]. This is also proved by the plot of Fig.5b. The experimental conditions of this plot are similar to the ones of Fig.5a, with the only exception that the observation time is 5 times shorter and here it is equal to 3.5 minutes after the increase of the PRT modulation current. The dependence of $\Delta \mathbf{T}[\mathbf{1 , 2}]$ on the temperature bias between the two thermistors $\mathbf{T}[\mathbf{1 , 2}]$ is also approximately linear, but the slope in this case is only $0.144 \%$ (when the scales in both axes are the same). It means that the nonlinearity of the material is dramatically changing in the process of interaction with the fields, decreasing by about 8.7 times in the presented comparison. The time interval, chosen as a reference in Fig.5b, corresponds to the observation point when the radiated power changes rapidly in time. So, it is natural that the uncertainty in case of Fig. $5 \mathrm{~b}$ is much larger $(\sim 5.3 \mu \mathrm{K})$ than that in case of Fig.5a (in which the temperature is close to its maximum value).

The nonlinear character of the response of the TSE to the modulation signal and the evident delay in time to the square-wave modulation are the typical features of the hysteresis effect in ferromagnetic and ferroelectric materials. Using this analogy, the hysteresis loops corresponding to the surface energy $\Delta \mathbf{T}[\mathbf{1}, \mathbf{2}]$ can be presented. The comparison of the slopes of the thermistor records in Fig. 2 shows that during the heating period of the cycle the excessive energy flux is propagating in the direction of the thermistor-1, while at the cooling period the excessive energy flux is observed in the direction of the thermistor-2. But in the material with absorption, the change of the direction of the energy flux corresponds to the change of the direction of the force acting on the unit volume of the field-particle system inside this material [24]. Thus, the thermal evolution process in case of the thermal surface energy is similar in many respects to the evolution process in ferroelectric materials, when the applied electrical field and the force on particles inside the material are periodically changing their directions in order to observe the hysteresis curve. So, in accordance with [16], in order to plot the hysteresis curve in case of the surface energy, it is necessary to change the direction of time when the energy flux inside the material changes its direction to the opposite one. The corresponding hysteresis curves are shown in Figs.6a-6c. The effect of the external heat source on the thermal evolution process and on the form of the TSE hysteresis loop will be clearly observed on the presented plots.

Here, we are to note that there is some difference in presentation of the hysteresis loops relative to the plots in [16]. The plots of Figs. 6a-6c here keep the information about all of the time intervals between the adjacent data points during the whole modulation cycle; so the cooling period is shown to be three times longer than the heating period.

In Fig.6a, the data-points of the heating period (between arrows $\mathrm{A}$ and $\mathrm{B}$ ) are presented by dots, while the points of the cooling period of the cycle (between arrows B and D) are shown as rhombi. The last point of the previous cooling period is marked by letter A. All of the presented data points in Fig. 6a are measured relative to the 6-th order polynomial fit, which was calculated for the reference points of four adjacent modulation cycles. So, the difference (of about a few micro Kelvin between the last data points of the two consecutive cycles (that is the difference between the positions of the points $A$ and $D$ ) is much smaller than the dimension of the rhomb. Naturally, this difference cannot be discerned at scale of the plot, when the span of the loop is more than $2200 \mu \mathrm{K}$.

It is worth noting that the plots of Figs. $6 a, 6 b$ and $6 c$ corresponded to three independent experiments, in which the temperature differences between the positions of thermistors 1 and 2 were $-2.6 \mathrm{mK}, 16.2 \mathrm{mK}$ and $57.1 \mathrm{mK}$, respectively. Here again (as in Figs.4a-4c) we can clearly observe the time delay of about 1.5 minute, which is necessary for the propagation of the excessive heat flux to the location of the thermistor-1 after the reflection of the energy flux from the gauging surface of the block. So, even when the current in the PRT is switched from $5 \mathrm{~mA}$ to $1 \mathrm{~mA}$, the heating of the block surface in the vicinity of the thermistor- 1 is still continuing. Thus, the change of the direction of the excessive energy flux at the position of the thermistor-1 [17] occurs at the time instant, which is marked by letter C. Also, it has been shown experimentally earlier that both the energy flux and the thermal surface energy fall in a fast way with the increase of the distance from the boundary of the artifact (Fig. 5 in [19]). It means that the plots of Figs.6a-6c here describe the evolution of the thermal energy close to the thermistor- 1 , and the value of the surface energy at the location of the thermistor-2 is quite negligible.
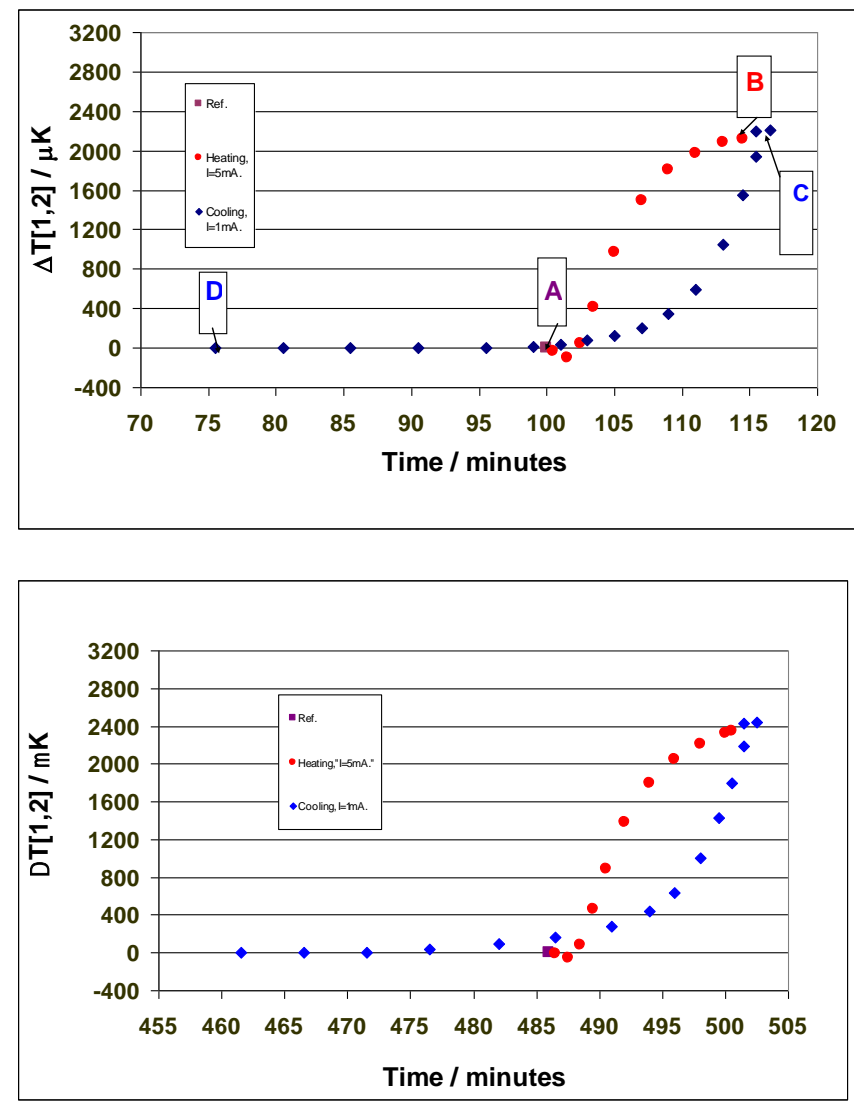


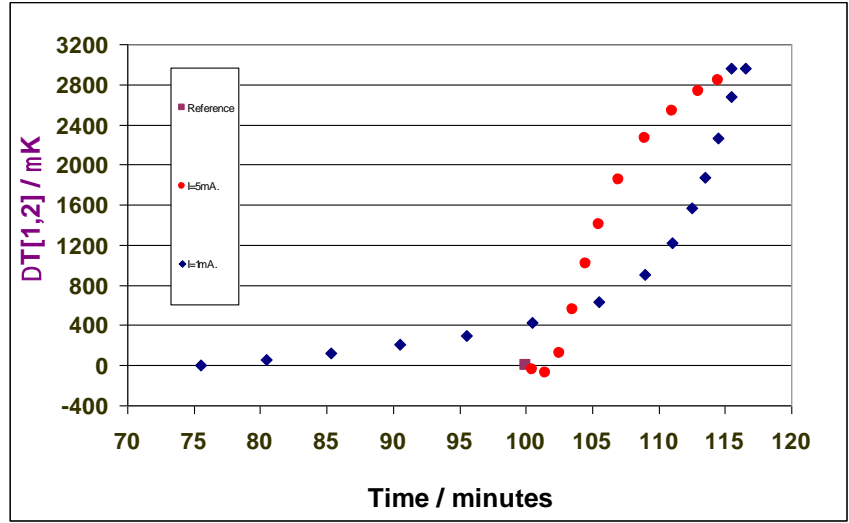

Figs.6a-6c. The effect of the temperature difference $\mathbf{T}[\mathbf{1}, \mathbf{2}]$ between the locations of the thermistors 1 and 2 on the form of the thermal hysteresis curves. The temperature differences $\mathbf{T}[\mathbf{1 , 2}]$ were: $-2.9 \mathrm{mK}$ (Fig.6a), $16.2 \mathrm{mK}$ (Fig.6b), and $57.1 \mathrm{mK}$ (Fig.6c), respectively.

To characterize quantitatively the dependence in time of the surface energy during the cooling period, we choose the point, which is observed on the plot 15 minutes later than the point C. For this point of the dependence of the surface energy as a function of time, the quantity $\Delta \mathbf{T}[\mathbf{1 , 2}]$ is equal to $44.8 \mu \mathrm{K}$; its ratio $(\mathrm{R})$ to the corresponding maximum value of $\Delta \mathbf{T}[\mathbf{1}, \mathbf{2}]$ (which in this case is $2207 \mu \mathrm{K}$ ) is $2.03 \%$, only. This point of the time dependence will be used when studying the effect of the auxiliary energy source on this process.

To demonstrate that effect, in Fig.6b the similar hysteresis loop is presented when the temperature bias $\mathbf{T}[\mathbf{1 , 2}]$ of $16.2 \mathrm{mK}$ was realized between the thermistor positions. In this case, the flux of energy, which is produced in advance by an auxiliary energy source, is directed from thermistor- 1 to thermistor-2. The magnitude of the surface energy in the selected point of the time dependence is now equal to $159 \mu \mathrm{K}$, and the value of $\mathrm{R}$ is increasing to $5.4 \%$. In Fig. $6 \mathrm{c}$ the temperature bias $\mathbf{T}[\mathbf{1 , 2}]$ between the thermistor positions is increased further to $57.1 \mathrm{mK}$. In this case, the TSE magnitude at the specified time moment increases to $214 \mu \mathrm{K}$, and its ratio to the corresponding maximum value of reaches $13.8 \%$. So, from the variations of forms of the hysteresis loops, we conclude that in relative units the cooling process becomes longer with the increase of the temperature bias $\mathbf{T}[\mathbf{1}, \mathbf{2}]$. Thus, the plots of Figs. 6a-6c demonstrate the effect of the auxiliary energy sources on the time dependence, on the form, and on the magnitude of the hysteresis loop. We are to remind here that the quantity $\Delta \mathbf{T}[\mathbf{1}, \mathbf{2}]$ describes the difference in the in the amounts of energies, which are radiated by the gauge block surfaces per unit time at positions of the thermistors 1 and 2; and this difference arises as a result of the periodic square-wave modulation of the PRT current.

As discussed above, the experimental results of Figs. $4 a-4 c$ and $6 a-6 c$ correspond to the case when the studies of the energy flux propagation process, which was stimulated by the increase of the modulation current in the PRT, were performed under the presence of the already existing energy flux between the locations of the thermistors that was produced by the auxiliary heat source. And for the studies of variations in time of the energy fluxes that are propagating inside a material artifact, synchronous measurements of the differential thermal velocities at two locations of this artifact are appropriate [18]. Indeed, in accordance with the Poynting theorem of Electrodynamics, the energy flux per unit time (described by the Poynting vector) through the boundary surface of the material artifact is defining the rate in time of the total energy variations within the volume of the artifact; where the total energy variations consist of the energy variations of EM field and the energy variations of the charged particles as a result of the work in unit time of electromagnetic forces on the charged particles within the indicated volume [25] (p.347). In the differential form this theorem of Electrodynamics states that the partial derivative in time of the total energy density of the field-particle system inside a material artifact plus the divergence of the Poynting vector is equal to zero ([25] p.348; [26] p.347). And from the thermal conduction theory [27], it follows that if the heat sources are not present inside the artifact, then the rate in time of the internal energy density of the field-particle system is defined by divergence of the energy current density of the field-particle system (eq.(52.9) in [27]). From this it follows (from the continuity of energy) that if a material object is in vacuum, then the energy, which is radiated into space per unit time from the unit area, is equal to the energy flux, which is delivered by the field-particle system to that area from inside the object (p. 166, eq.(52.14) in [27]), when, naturally, the reflection of the wave momentum of the field-particle system from the boundary [19] is taken into account. As the thermistors (as pointed out below) were calibrated on the surface of a long gauge block under the condition of thermal equilibrium (when temperature gradient along the block surface was negligible), so the readings of the thermistor channels corresponded to the flux of the radiated energy from the block surface, when that flux was expressed in terms of the thermodynamic temperature (in accordance with Stefan-Boltzmann law). As a result of a complete symmetry of our experimental set-up in $\mathrm{x}-$, and $\mathrm{y}$ - directions, the difference in the readings of the thermistor channels can arise only due to the difference in the fluxes of energies at the locations of the thermistors in $\mathrm{z}$-direction (along the line perpendicular to the gauging surfaces). So, as the quantity $\Delta \mathbf{T}[\mathbf{1 , 2}]$ describes the difference in the fluxes of energies in z-direction, the synchronously measured difference in thermal velocities, $\Delta \mathbf{V}[\mathbf{1 , 2}]$, is a useful vector quantity, which characterizes the rate in time of this difference in energy fluxes and, consequently, is of primary importance in the description of the evolution process. It is worth noting here that that when there are no energy sources inside the material object, the expression for the energy current density of the field-particle system in the energy continuity relation can be presented as a product of the total energy density of the field-particle system and the velocity of the energy propagation in the direction of the maximum flux density (eq. (4.16 in [24]; pp.346-349 in [26]). Using this relation, the effective velocity of the energy propagation in a steel gauge block was measured to be about $15 \mathrm{~mm} /$ minute [21]. So, the time responses of thermistors and multi-meters are sufficient for the studies of time dependences of the energy fluxes inside gauge block materials [17].

Realizing this idea, in Fig.7a the difference between the synchronously measured thermal velocities $\mathbf{V}[\mathbf{1 , 2}]$, which were recorded in the channels 1 and 2, is presented as a function of time (during one modulation cycle) under the experimental conditions of Fig.4a. Again, the data-points of 


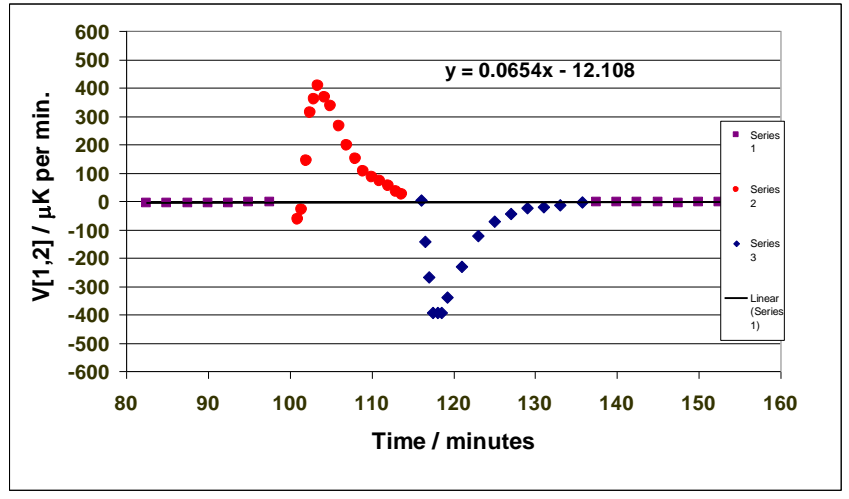

Fig.7a. Record of the thermal evolution process when characterized by the difference in thermal velocities $\Delta \mathbf{V}[\mathbf{1 , 2}]$ at the locations of thermistors No.1 and No.2. The record was obtained for the temperature difference between the channels $\mathbf{T}[1,2]$ equal to $-2.9 \mathrm{mK}$.

the heating period are presented by dots, and the points of the cooling period of the modulation cycle are shown by rhombi and squares (reference points). A linear fit to all of the reference points is shown in the figure as a bold line, and the corresponding equation of the fit is presented in the inset.

As it follows from the presented plot, the use of a relatively long cooling period, which is three times longer than the heating period, gives an opportunity to realize conditions when the difference between the thermal velocities in the thermistor channels is within a few $\mu \mathrm{K} /$ minute for the reference points, and the standard deviation of all of the reference points relative to the linear fit is about a couple of $\mu \mathrm{K} /$ minute. First, it means that about 30 minutes after the beginning of the cooling period of the modulation cycle the energy fluxes at the locations of the thermistors are equal. Second, the quantity $\Delta \mathbf{T}[\mathbf{1 , 2}]$ (describing the effect of power modulation by the PRT) is practically equal to zero at the end of the cooling period (in agreement with Fig.6a), so that the length of the cooling period was properly selected. Third, the signal-to-noise ratio in the differential measurements of thermal velocities is quite high, as the peak value of the effect of current modulation in the PRT exceeds $400 \mu \mathrm{K} /$ minute, and the standard deviation for the reference points is about $2-3 \mu \mathrm{K} /$ minute.

The polynomial fit to reference points of several modulation cycles was usually used as a reference function, relative to which all the variations of the experimental quantity $\mathbf{V}[\mathbf{1 , 2}]$ were measured in order to obtain the induced variations $\Delta \mathbf{V}[\mathbf{1 , 2}]$ of the difference in thermal velocities in channels 1 and 2 as a function of time. As from the linear equation of the fit in Fig.7a, we find that for the duration of the heating period (15 minutes) the variation of the reference function is within $1 \mu \mathrm{K} /$ minute, so it is quite natural that under the selected experimental conditions, the choice of the reference function (linear or polynomial) has very small effect on the result of synchronous measurements of the quantity $\Delta \mathbf{V}[\mathbf{1 , 2}]$. Besides that, as the differences of the plotted reference function in Fig.7a (shown as a solid thicker line) are quite negligible relative to the abscissa axis of this plot, a separate presentation of the quantity $\Delta \mathbf{V}[\mathbf{1 , 2}]$ versus time on a new figure is not reasonable.

As it follows from Fig.7a, during most of the time in the heating period the quantity $\Delta \mathbf{V}[\mathbf{1 , 2}]$ is positive, and during the cooling period it is mainly negative. The important result following the plot of Fig.7a (which can be specially emphasized) is that the excessive energy fluxes of reasonable amounts exist only during a relatively short time (of about 15-16 minutes) after the change of the PRT modulation current. This time dependence is quite complicated: the excessive flux in a few minutes reaches its maximum value, and then there is a gradual decrease of its magnitude. From the experimental data points of Fig.7a, we conclude that the maximum value, $\Delta \mathbf{V}_{\text {max }}$, of the recorded velocity difference $\Delta \mathbf{V}[\mathbf{1 , 2}]$ in this experiment was $\sim 411.2 \mu \mathrm{K} /$ minute, and it was observed at the time interval of 3.5 minutes after the increase of the current in the PRT. The maximum value of the quantity $\Delta \mathbf{V}[\mathbf{1 , 2}]$ is the key point on this plot and we shall check below how it can be influenced by the change of the parameters of the experiment. The other point on the plot during the heating period, which can be used to characterize the time dependence of the thermal process, will correspond to the time interval $\tau$ of 14 minutes after the beginning of the modulation cycle, i.e., just one minute before the reduction of the current in the PRT. The corresponding quantity of the difference in temperature velocities $\Delta \mathbf{V}[\mathbf{1 , 2}]$ between the channels 1 and 2 for this value of $\tau$ was found to be $18.53 \mu \mathrm{K} /$ minute. The ratio of this quantity to the maximum velocity difference $\Delta \mathbf{V}_{\max }$ (denoted here by $\delta_{\mathrm{h}}$ ) is $4.51 \%$ for the temperature difference $\mathbf{T}[\mathbf{1 , 2}]$ of $-2.9 \mathrm{mK}$. We remind that the quantity $\mathbf{T}[\mathbf{1 , 2}]$ corresponds to the bias between the channels, which is produced by an auxiliary energy source and which is measured at the end of the cooling period of the modulation cycle.

As it follows from Fig.7a, during the cooling period the quantity $\Delta \mathbf{V}[\mathbf{1 , 2}]$ is becoming negative, changing its sign in comparison with the heating period. It means that the energy, which is radiated by the block surface per unit time, is larger in the vicinity of the thermistor-2, and consequently, the flux of energy, which is delivered by the field-particle system to the correspondent volume, is also larger. It should be noted here, that the time dependence for the cooling period cannot be presented as an inversion of the plot of the heating period relative to abscissa axis. Besides, it will be shown below that the effect of the auxiliary energy sources is somewhat different for these two periods of the thermal evolution process.

To demonstrate this, we characterize the cooling period of the cycle by the minimum value $\left(\Delta \mathbf{V}_{\min }\right)$ of the quantity $\Delta \mathbf{V}[\mathbf{1 , 2}]$ and by the value of the quantity $\Delta \mathbf{V}[\mathbf{1 , 2}]$ that corresponds to the time interval $\tau$ of 29 minutes after the increase of the modulation current in the PRT, or 14 minutes after the decrease of the modulation current. In this case the comparison of the processes for the heating and cooling periods is easy to perform. For the temperature difference $\mathbf{T}[1,2]$ of $-2.9 \mathrm{mK}$, the quantity $\Delta \mathbf{V}_{\text {min }}$ (Fig.7a) is equal to $-392.7 \mu \mathrm{K} /$ minute. So, the ratio $\Delta$ of the absolute value of $\Delta \mathbf{V}_{\text {min }}$ to the maximum value $\Delta \mathbf{V}_{\max }$ is about $95.5 \%$. And in accordance with our measurements, the quantity $\Delta \mathbf{V}_{\text {min }}$ is observed in the time interval $\tau$ of about 3.1-3.2 minutes after the decrease of the modulation current in the PRT. (For the maximum value of $\Delta \mathbf{V}[\mathbf{1 , 2}]$ the corresponding time is about 3.5 minutes after the increase of the current in the PRT.)

To demonstrate the effect of the additional external heat source on the evolution process, in Figs.7b and $7 \mathrm{c}$ the time dependences of the quantity $\Delta \mathbf{V}[\mathbf{1 , 2}]$ are presented for the temperature differences $\mathbf{T}[\mathbf{1 , 2}]$ of $16.2 \mathrm{mK}$ and $57.1 \mathrm{mK}$, 


\section{Experimental Demonstration of the Relational Character of Time in Natural Processes; Concept of Time in the Relational World}

respectively. The positive values of $\mathbf{T}[\mathbf{1 , 2}]$ mean that these series of measurements were performed when the heat source No. 2 in Fig.1 was switched on. As the distribution of temperature in a well thermally-isolated homogeneous gauge block is a non-linear function of the displacement of a thermometer along the length of a gauge block [14] (Fig. 8), the activation of the heat source No.2 produced a much larger temperature difference $\mathbf{T}[\mathbf{1}, \mathbf{2}]$ between the positions of the thermistors 1 and 2 than the activation of the heat source No.1 (for the same level of dissipated power). So, the relatively large temperature difference of $57.1 \mathrm{mK}$ was obtained without problems: even under the indicated conditions, the velocity difference $\Delta \mathbf{V}[\mathbf{1}, \mathbf{2}]$ at the end of the cooling period was within a few $\mu \mathrm{K} /$ minute, and consequently, the precise synchronous temperature measurements were realizable. From the comparison of the dependences in Figs.7a-7c it follows that the auxiliary energy source changes the thermal evolution process (induced by the modulation of the output power in the PRT) in many aspects.
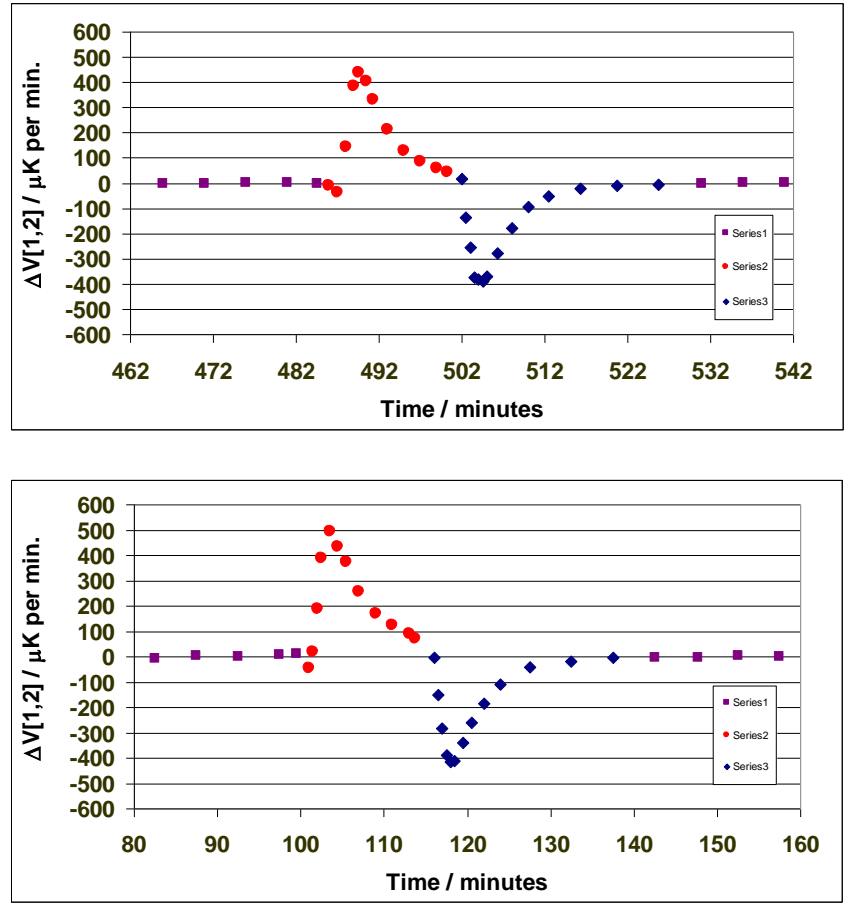

Fig.7b-7c. Records of the difference in thermal velocities $\Delta \mathbf{V}[\mathbf{1 , 2}]$ at the locations of thermistors No.1 and No.2. The records were obtained for the temperature differences between the channels T[1,2] equal to $16.2 \mathrm{mK}$ (Fig.7b) and $57.1 \mathrm{mK}$ (Fig.7c), respectively.

First, the excessive energy flux, which is delivered by the field-particle system toward the unit volumes in the vicinity of the gauging surface and which variations in time can be described by the quantity $\Delta \mathbf{V}[\mathbf{1 , 2}]$, is steadily growing with the increase of the quantity $\mathbf{T}[\mathbf{1 , 2}]$. For example, in explicit way, the dependence of the maximum value $\Delta \mathbf{V}_{\max }$ of the quantity $\Delta \mathbf{V}[\mathbf{1 , 2}]$ as a function of the temperature bias $\mathbf{T}[\mathbf{1 , 2}]$ is presented in Fig.8. The dependence of $\Delta \mathbf{V}_{\text {max }}$ in the range of $411 \mu \mathrm{K} /$ minute to $493 \mu \mathrm{K} /$ minute is described by a linear function of the parameter $\mathbf{T}[\mathbf{1 , 2}]$ with a very high precision. The equation of the linear regression line (describing the dependence of the quantity $\Delta \mathbf{V}_{\max }$ on the temperature difference $\mathbf{T}[\mathbf{1 , 2}]$ ) is presented in the inset of Fig.8. For the results of three independent experiments shown in Fig.8, the maximum deviation of the data points from the regression line is less than $0.12 \mu \mathrm{K} /$ minute. This means that the presentation of $\Delta \mathbf{V}_{\max }$ with four digits has some grounds. It also means that $\mathbf{T}[\mathbf{1 , 2}]$ is a good parameter for the description of the thermal evolution process, as the experimental conditions in the discussed case correspond to a wide range of temperature gradients in the measured regions of the gauge block, and they cover both directions of the energy fluxes produced by the auxiliary heat sources.

The plot of Fig. 8 is illustrating the influence of an auxiliary energy source on the maximum value of the quantity $\Delta \mathbf{V}[\mathbf{1}, \mathbf{2}]$, which is observed at 3.5 minutes after the increase of the modulation current in the PRT. To demonstrate quantitatively the effect of the external energy sources on the time dependence of the evolution process during the whole heating period of the modulation cycle the plots of Figs. 9 and 10 are presented. In Fig.9 the quantity $\Delta \mathbf{V}[\mathbf{1 , 2}]$ is plotted as a function of $\mathbf{T}[\mathbf{1 , 2}]$ (similar to the plot of Fig.8), but the measurements of $\Delta \mathbf{V}[\mathbf{1}, \mathbf{2}]$ are performed for the time interval $\tau$ of 14 minutes after the increase of the modulation current and i.e., 1 minute before the decrease of the modulation current in the PRT.

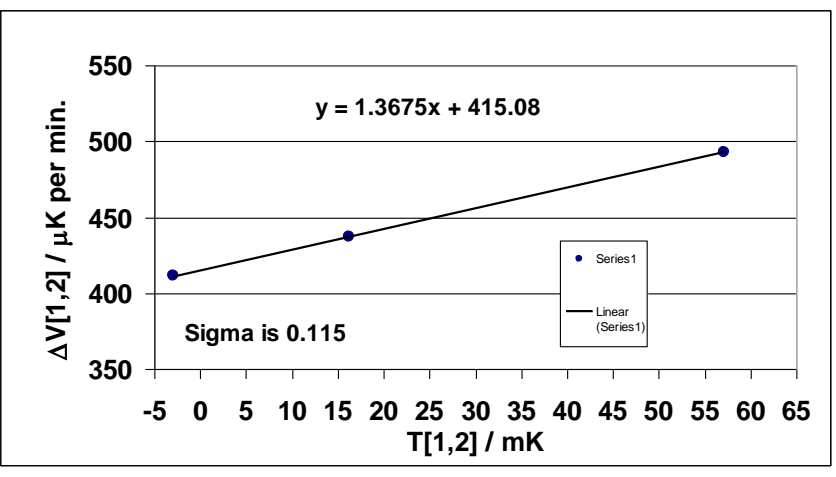

Fig.8. The dependence of the maximum value of the difference in the thermal velocities $\Delta \mathbf{V}[\mathbf{1 , 2}]$ on the temperature difference between the channels $\mathbf{T}[\mathbf{1}, \mathbf{2}]$.

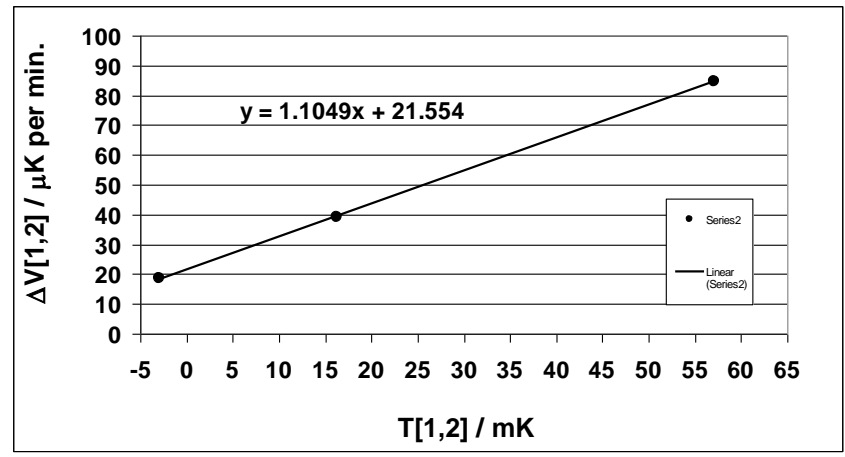

Fig.9. The dependence of the quantity $\Delta \mathbf{V}[\mathbf{1 , 2}]$, which is corresponding to the time interval of 14 minutes after the beginning of the modulation cycle, on the temperature difference between the channels $\mathbf{T}[\mathbf{1}, \mathbf{2}]$.

From Fig.9 we conclude that in the whole investigated temperature range of $\mathbf{T}[\mathbf{1 , 2}]$ the dependence of $\Delta \mathbf{V}[\mathbf{1 , 2}]$ can be considered again as linear: the standard variation of the data points $(0.23 \mu \mathrm{K} /$ minute $)$ is much smaller than the observed variation of $\Delta \mathbf{V}[\mathbf{1 , 2}](66 \mu \mathrm{K} /$ minute). But in contrast to the plot of Fig.8, the magnitude of the measured quantity $\mathbf{\Delta} \mathbf{V}[\mathbf{1 , 2}]$ has dropped by more than 19 times (at 
$\mathbf{T}[\mathbf{1 , 2}]=0$ ), while the slope of the dependence has also decreased, but only by about $23 \%$ relative to the slope of Fig.8.

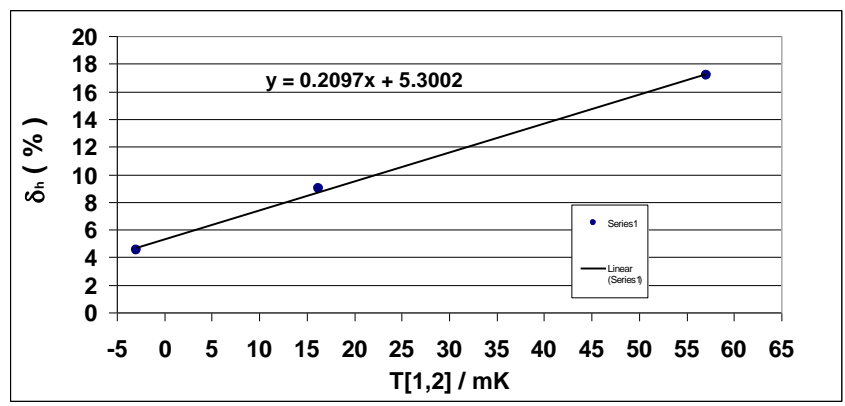

Fig.10. The dependence of the quantity $\delta_{\mathrm{h}}$ on the temperature difference between the channels $\mathbf{T}[\mathbf{1 , 2}]$. (See text for other details).

The last comparison corresponds to the case when the effect of the temperature bias is related to the variations of $\Delta \mathbf{V}[\mathbf{1 , 2}]$, which are measured in absolute values (i.e., in micro Kelvin per minute). But for the description of the form of the time dependence is more appropriate to use relative units. So, in Fig.10 a dimensionless quantity $\delta_{\mathrm{h}}$ is presented as a function of $\mathbf{T}[\mathbf{1 , 2}]$. Here, $\delta_{\mathrm{h}}$ is defined as a ratio of the value of $\Delta \mathbf{V}[\mathbf{1 , 2}]$ at $\tau$ equal to 14 minutes to the maximum value $\Delta \mathbf{V}_{\text {max }}$, when both values are measured, naturally, at the same temperature bias $\mathbf{T}[\mathbf{1}, \mathbf{2}]$.

The dependence of the quantity $\delta_{\mathrm{h}}$ on the temperature difference $\mathbf{T}[\mathbf{1}, \mathbf{2}]$ can also be described as a linear function; for the three independent experiments presented in Fig.10, the value of the ratio $\delta_{\mathrm{h}}$ is changing from $4.5 \%$ to $17.2 \%$, while the maximum deviation of the points relative to the fit is less than $0.28 \%$. From the plot of Fig. 10 we can conclude that as the ratio $\delta_{\mathrm{h}}$ increases by more than 3.8 times for the increase of the temperature bias $\mathbf{T}[\mathbf{1 , 2}]$ from $-2.9 \mathrm{mK}$ to $57,1 \mathrm{mK}$. It means that the duration of the existence of the excessive energy flux (described by the quantity $\Delta \mathbf{T}[\mathbf{1 , 2}])$ depends on the direction and on the magnitude of the energy flux, which has been created in the material object in advance by the auxiliary, external energy source. Indeed, in this experiment the time interval $\tau$ after reaching the maximum value of $\Delta \mathbf{V}[\mathbf{1 , 2}]$ is fixed, and the quantity $\delta_{\mathrm{h}}$ is normalized on the magnitude of $\Delta \mathbf{V}_{\text {max }}$; consequently, the process becomes slower with the increase of temperature bias. And it is not that difficult to foresee that the thermal evolution process cannot be described in principle [19] by any "effective theory" (p.108 in [2]) that is based on Newtonian paradigm.

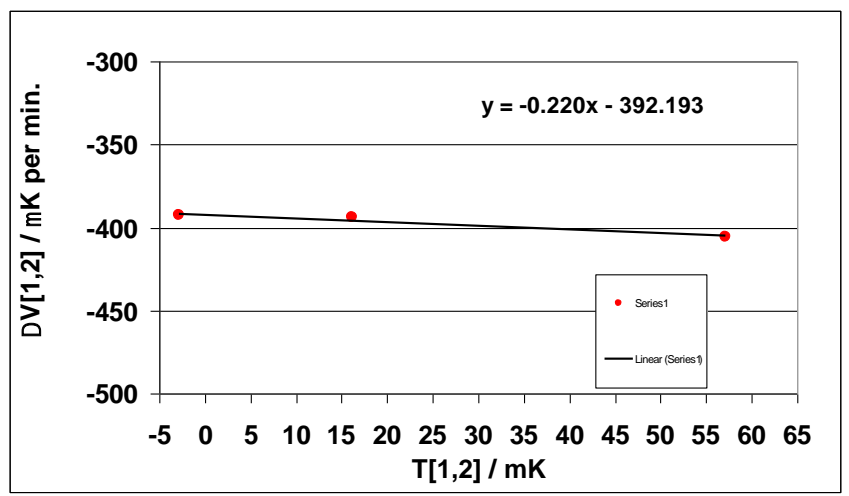

Fig.11. The dependence of the minimum values $\Delta \mathbf{V}_{\text {min }}$ of the quantity $\Delta \mathbf{V}[\mathbf{1 , 2}]$, which are is observed during the cooling period of the modulation cycle, on the temperature difference between the channels $\mathbf{T}[\mathbf{1 , 2}]$.

To demonstrate further the differences between the processes during the heating and during the cooling periods of the modulation cycle, the corresponding dependences for the cooling period are shown in Figs.11-14, so that the comparisons can be performed quantitatively, using the parameters that were introduced in the discussions of Fig.7a. First, the minimum values $\Delta \mathbf{V}_{\text {min }}$ of the quantity $\Delta V[\mathbf{1}, \mathbf{2}]$, which are clearly observed on the experimental dependences of Figs.7a-7c during the cooling period, are to be compared with the maximum values $\Delta \mathbf{V}_{\mathbf{m a x}}$, which are recorded during the heating periods of the modulation cycle. The dependence $\Delta \mathbf{V}_{\text {min }}$ as a function of the vector quantity $\mathbf{T}[\mathbf{1 , 2}]$ is presented in Fig.11 together with the equation of the corresponding linear fit.

It is clear that the absolute value of $\Delta \mathbf{V}_{\text {min }}$ (similar to $\Delta \mathbf{V}_{\text {max }}$ ) is increasing with the increase of the quantity $T[\mathbf{1 , 2}]$, but the effect of the external energy source is 6.2 times smaller than in case of the plot of Fig. 8 (as it follows from the comparison of the equations in Figs. 11 and 8). It means that the evolution processes during the heating and cooling periods of the modulation cycle are quite different.

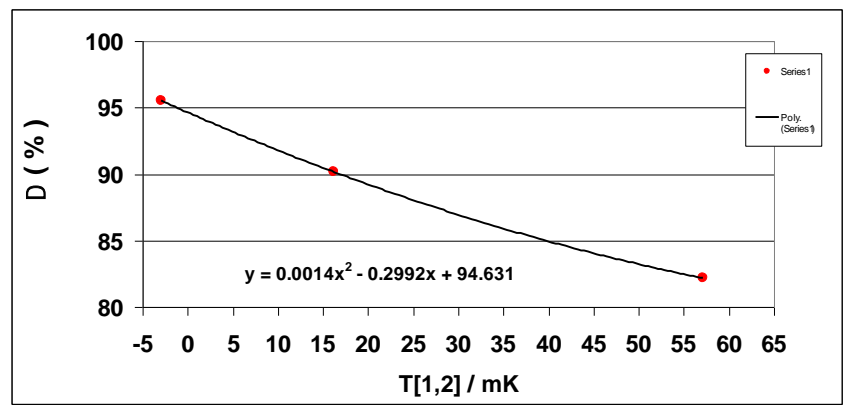

Fig.12. The ratio $\Delta$ of the absolute value of $\Delta V_{\text {min }}$ to $\Delta V_{\text {max }}$ is presented as a function of the temperature difference between the channels $\mathbf{T}[\mathbf{1 , 2}]$.

Even more obvious confirmation of this statement is given by the plot of Fig.12. Here, the quantity $\Delta$, which was defined earlier by the ratio the modulus of $\Delta \mathbf{V}_{\text {min }}$ to the corresponding maximum value $\Delta \mathbf{V}_{\text {max }}$, is presented as a function of the temperature difference $\mathbf{T}[\mathbf{1 , 2}]$. With the increase of the temperature bias from $-2.9 \mathrm{mK}$ to $57.1 \mathrm{mK}$, the quantity $\Delta$ is decreasing nonlinearly from $95.5 \%$ to $82.2 \%$. The other, earlier selected point on the time dependence, which was used to characterize the cooling period, corresponded to the time interval $\tau$, which was equal to 14 minutes after the decrease of the modulation current in the PRT. The corresponding dependence of the quantity $\Delta V[\mathbf{1 , 2}]$ at this $\tau$ as a function of $\mathbf{T}[\mathbf{1 , 2}]$ is shown in Fig.13, and it can be directly compared with the corresponding dependence of Fig.9. 


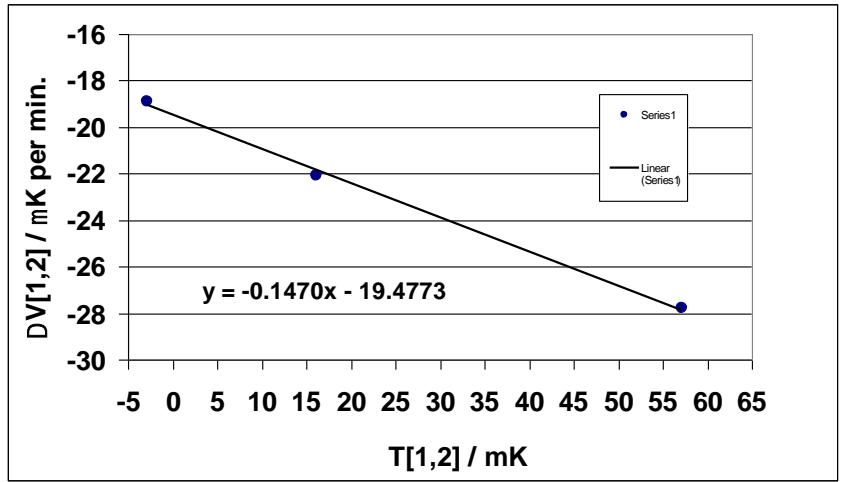

Fig.13. The dependence of the quantity $\Delta \mathbf{V}$, which is measured at the time interval $\tau$ equal to 14 minutes after the decrease of the modulation current, is shown as a function of the temperature difference between the channels $\mathbf{T}[\mathbf{1 , 2}]$.

In accordance with the plots of Figs.7a-7c, all the values in Fig.13 are negative and are describing the excessive cooling of the elementary volumes of the block in the vicinity of thermistor- 1 . In the presented range of the temperature bias from $-2.9 \mathrm{mK}$ to $57.1 \mathrm{mK}$, the corresponding variation of the quantity $\Delta \mathbf{V}[\mathbf{1 , 2}]$ occurs in the range between $-18.9 \mu \mathrm{K} /$ minute and $-27.8 \mu \mathrm{K} /$ minute. The maximum deviation of the experimental points relative to the linear fit is less than $0.22 \mu \mathrm{K} /$ minute. The increase of the temperature difference $\mathbf{T}[\mathbf{1 , 2}]$ of $60 \mathrm{mK}$ results in the increase of the absolute value of the quantity $\Delta \mathbf{V}[\mathbf{1 , 2 ]}$ in Fig.13 by about $47 \%$. This should be compared with the corresponding effect of the external energy source on the quantity $\Delta \mathbf{V}[\mathbf{1 , 2}]$ during the heating period (Fig.9), where the corresponding increase of $\Delta \mathbf{V}[\mathbf{1 , 2}]$ was $457 \%$ for exactly the same temperature difference.

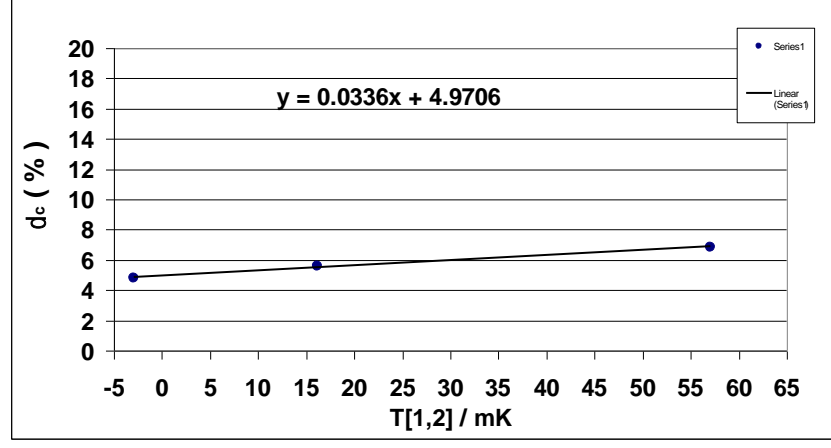

Fig.14. The dependence of the quantity $\delta_{\mathrm{c}}$ on the temperature difference between the channels $\mathbf{T}[\mathbf{1 , 2}]$. (See text for other details).

To receive some quantitative information about the time dependence of the excessive cooling process, it is more convenient to use relative measurements. For this purpose, in Fig.14 the ratio $\delta_{c}$ of the quantity $\Delta \mathbf{V}[1,2]$ at $\tau$, which is equal to 14 minutes after the beginning of the cooling period, to the minimum value of $\Delta \mathbf{V}_{\min }$ is presented as a function of temperature difference $\mathbf{T}[\mathbf{1 , 2}]$. The increase of the temperature difference from $-2.9 \mathrm{mK}$ to $57.1 \mathrm{mK}$ results in the increase of $\delta_{\mathrm{c}}$ from $4.87 \%$ to $6.85 \%$. The maximum deviation of the experimental points relative to the linear fit is less than $0.09 \%$ in this case. When comparing the slopes of the dependences of the dimensionless quantities $\delta_{\mathrm{h}}$ and $\delta_{\mathrm{c}}$ in Figs.
10 and 14, we find that the effect of the external energy source is 6.24 times larger for the heating period relative to the cooling period. Thus, the relative measurements, performed in the form of $\delta_{c}$, and an absolute type of measurements (illustrated by Fig.13), both clearly show that the cooling process in the vicinity of the gauging surface slows down with the increase of the temperature bias $\mathbf{T}[\mathbf{1 , 2}]$ (as the magnitude of the negative quantity $\Delta \mathbf{V}[\mathbf{1 , 2}]$ subsides slower in time). But both, the time dependences and amplitude characteristics of the evolution process are influenced by an external source of radiation in a basically different way for the heating and for the cooling periods of the modulation cycle. These dependences cannot be described by mathematical relations, as in the evolution process there is a huge number of interacting bodies, which are continuously changing their properties in the process of interaction and are continuously producing the common EM field, so that all these objects are related to each other and, naturally, cannot be considered as independent [19].

\section{CONCLUSIONS AND DISCUSSIONS}

1. The experiments presented in the previous section show that the thermal evolution process, which is generated by the square-wave modulation of the flux of EM energy to the material object, is modified by the presence of the other external energy source that produces an additional energy flux inside the material artifact. And this energy flux inside the artifact can be precisely detected at the end of the cooling period. It means that another experimental confirmation is given to the fundamental observation that the principle of superposition of the EM fields is not valid for the processes, which occur inside and outside the material objects [16-18]. In particular, it is demonstrated here that the thermal process, characterized by the surface energy $\Delta \mathbf{T}[\mathbf{1 , 2}]$, evolves slower in time with the increase of the temperature bias $\mathbf{T}[\mathbf{1}, \mathbf{2}]$, which is produced by the auxiliary energy source. The other important experimental observation, which states that the results of the differential temperature measurements depend crucially on the time interval after the application of the flux of energy, directly indicates that the property of the material object to radiate EM energy from its surface is dependent on the indicated time interval. And this time dependence is described by the hysteresis effect with the well-defined hysteresis loop (Figs. 6a-6c). But it has been demonstrated earlier that the hysteresis curve and the radiated energy depend critically on the position of the source of the modulated energy flux relative to the surface of the material artifact $[15,16]$, and on the position of the energy emitting surface relative to the gauging surface $[19,21]$. Thus, every part of material object, which is irradiated by a flux of energy, contributes to the emission of the broad-band EM field with a specific time delay $[17,18]$; the intensity of this radiation varies continuously in space along the surface of the material object $[19,20]$, and the rate of the emission varies in time, in accordance with the present study. But it has been shown experimentally that in case of the interaction of EM radiation with material artifacts, the physical factors, which are responsible for the changes of the properties and of the structure of a material objects, are the fluxes of energy and momentum (described by the Poynting vector) through the boundary surface of this artifact $[17,16]$. Here it is worth 
noting, that the very concept of the surface energy is intrinsically related to the fluxes of energy and of momentum of the field-particle system inside the artifact [17] and their reflection from the boundary [20]. And these propagating energy fluxes can be easily detected by the amount of EM energy, which is radiated by different parts of the artifact surface into the outer space. The energy spectrum of this radiation is inevitably different from the spectrum of the incident EM field. Indeed, the temperature of the heat source (producing the required EM radiation) is always higher than the temperature of the material artifact in all our experiments. Besides that, the flux of radiated energy (and temperature of the surface) of the studied material object is continuously changing in time and along length of the block in response to the applied modulation of the magnitude of the Poynting vector [20,21]. Thus, from the results of our experimental studies, it can be inferred that in the presence of a source of the external flux of energy (the Sun, for example), for every material object on the Earth there are infinite number of secondary sources of EM radiation, whose mutual interaction process defines the parameters of the resulting Poynting vector that is acting on the specified material object. Recalling that the superposition principal is not valid for EM fields [16], we coming to the conclusion that for any specified material artifact, there are always a huge number of material objects in the Universe that are participating (through their own evolution process) in the creation of the common EM field and the resulting Poynting vector for the specified artifact. And, as the flux of the Poynting vector through the surface of the material object is the source of the following changes in the properties and the structure of the object [19], it follows from our experimental studies that the evolution process in a particular artifact is related to evolution processes in all material objects of the Universe through the flux of the common Poynting vector. And it has been proved experimentally that the external conditions, which are directly responsible for the creation of the resulting Poynting vector, play an absolutely crucial role in the evolution processes in material artifacts. For example, this relation becomes obvious from comparisons of the plots in Figs. 2 and 5 in [19] with the plots in Figs. 2a, 3 and 5 in [21]. From these studies it follows that in a steel $100 \mathrm{~mm}$ gauge block, which is located in a Dewar system, the oriented motion of the field-particle system disappears completely at the distance of about 30mm (Fig.5 in [19]); meanwhile, inside a homogeneous temperature field of the unique Kösters interferometer, the oriented motion can be traced along the distances of more than $1000 \mathrm{~mm}$ in a long $(900 \mathrm{~mm})$ steel gauge block (Figs. 2a and 5 in [21]).

And the relational character of coexistence of material objects in the Universe, Gottfried Wilhelm Leibniz called in the paragraph 59 of his "Monadology" [5] that "universal harmony, according to which every substance exactly expresses all others through the relations it has with them." Further development of this idea can be found in $\$ 87$ of the fifth Leibniz's letter to Dr. Clarke [4a], where he writes, "every simple Substance_is by its nature a concentration and a living mirror of the whole Universe, according to its Point of view," or its "perspective on the Universe", as it is further explained in [5] (\$57).

In this quotation, special attention should be paid to the last part of the statement, which is in deep agreement with the results of our experiments. Indeed, as the Poynting vector of EM field is inevitably changing in the Universe from one point of space to the other, and as the flux of the Poynting vector through the artifact surface in accordance with $[17,19]$ defines the "fine" properties and the "fine" structure of the material object (including not only the property to radiate EM field, but also including the amounts of stresses and of deformations, of the mass and of the charge transfer inside the material), then the properties and the structure of a particular object are defined also by its position in the Universe. Consequently, it follows directly from the results of our experiments that the existence of two identical objects in the Universe is impossible. And this is the second fundamental principle of Leibniz Natural Philosophy that he called the Identity of Indiscernibles in his fourth letter to Dr. Clarke [4b]. In paragraph 4 of that letter Leibniz explains, "There is no such thing as Two Individuals indiscernible from each other." And $\$ 6$ in [4b] states, "To suppose two things indiscernible, is to suppose the same thing under two Names." The views of Leibniz on the Natural Philosophy are expressed in $\$ 5$ in [4b], where we find: "Those great Principles of a sufficient Reason, and of the Identity of Indiscernibles, change the State of Metaphysicks. That Science becomes real and demonstrative by means of these Principles; whereas before, it did generally consist in empty Words."

It should be specially emphasized here that the second great principle of the Leibniz's Natural Philosophy, the Identity of Indiscernibles, is in agreement not only with the presented experimental studies, but it has been confirmed earlier by the infinite number of experimental observations in Biology, Zoology, Palaeontology and Astronomy.

2. The topics discussed in the previous item are closely related to the conclusion, which is obtained from the analysis of only experimental results, that the mathematical description of processes in Nature is basically impossible [19]. The physical reasons for that are the violations of symmetries in time and in space in natural processes that are superimposed on the invalidity of the superposition principle and the infinite number of interacting bodies. As it is emphasized in [16,17], the basic properties of the thermal evolution process - the lack of symmetries (in space and in time) and the infinite number of influence parameters - are in agreement with the studies performed in different areas of Natural sciences. In astronomy, for example, the concept of the arrow of time (or irreversible time) was introduced in 1927 by the prominent English astrophysicist and astronomer Arthur Eddington. And this distinguished direction of time (or arrow of time), according to A. Eddington, was determined also by the studies of organizations of material objects in the Universe. It was Arthur Eddington, the leader of the team of British astronomers, who was able (during the total eclipse on May 29, 1919) to perform the first test of the General theory of Relativity and to measure precisely extremely slight bending of light around the Sun (or gravitational lensing effect, as it is called nowadays). On November 6, of the year 1919 A. Eddington announced the results of his measurements. When using K. Popper - A. Einstein approach to Natural sciences, we can conclude that A. Eddington by that report presented, for the first time, the quantitative refutation of the whole Newtonian theory (including, naturally, the Newtonian concept of time) and 
gave simultaneously the first experimental support to the General Relativity theory of A. Einstein.

By the year 1927, regular astronomical observations of the Earth's rotation, which were started in the year 1900, gave the astronomers sufficient experimental data to make a conclusion about the time-irreversible character of the motion of our planet. In the early fifties, this astronomical discovery was directly confirmed by the corresponding comparisons with the newly-developed quantum clocks. Later, with the use of new techniques in astronomical observations of the Earth's rotation (such as radio-astronomy and laser ranging), the astronomers came to the conclusion of the infinite number of influence factors that are affecting the rotation process of our planet, so that the corresponding precise theoretical predictions are basically impossible [28].

In Zoology, the time irreversible character of changes in Nature was known much earlier; the year 1858 is associated with the discovery of the Natural evolution theory by $\mathrm{Ch}$. Darwin and A. R. Wallace, when they published the paper [29] entitled, "The Tendencies of Species to Form Varieties, and the Perpetuation of Varieties and Species by Natural Means of Selection". The asymmetry in space also follows from experimental studies of different types of hysteresis effect in physics [19]. The numerous examples of the asymmetry in space are known from Molecular Biology, and some of them can be found nowadays even in the text-books on physics [3c] The asymmetry in space has been established even in the properties of the thermal radiation in Universe with the help of new radio-telescopes [30] by G. Smoot, M. Gorenstein \& R.A. Muller in (1977). But specially, it is worthy of note that the time is irreversible in the well-known ferromagnetic, ferroelectric hysteresis effects and in the thermal evolution process, where the influence on the system under study and the detection of the variation of the state of the system are performed through electro-magnetic interactions, only. The irreversible time also follows from the standard procedures of melting and freezing of pure metals (that are used world-wide for realization of "thermodynamic" temperature), as the paths of the system are basically different for freezing and melting procedures [31] (Fig.3-7); and when these paths are combined in a single plot, they present a standard hysteresis loop. This means that all parts of theoretical physics, which are not able to demonstrate explicitly that the irreversible time and the hysteresis effect follow from solely electromagnetic type of interactions, are falsified by a huge number of experiments indicated above.

Meanwhile, in contrast to the presented experimental facts, the first law of Newton inevitably requires the homogeneity and the isotropy of time and of space [32], as otherwise, a description of the motion of a material object in an arbitrary direction with constant velocity cannot be realized. In Newtonian mechanics, the vector concepts of velocity, angular velocity, acceleration and angular acceleration, besides the homogeneity and isotropy of time and space, require also independency of spatial coordinates from the time coordinate.

The absolute impossibility to describe by the language of mathematics processes in Nature can be inferred from the experiments presented in this paper. Indeed, the thermal hysteresis effect (described in detail here) is only a tiny part of the thermal evolution process, when the detection of the state of the system in the process of interaction of matter with the
EM field is performed only in two small areas of the material object. But in the case of the hysteresis effect, the adequate description of it is based on the simultaneous nonlinear interaction of, at least, six-seven material objects, which are constantly changing their properties in that interaction process. First, we have a system that consists of a gauge block with three thermometers attached to its surface. One of these thermometers is used as a modulator, and two others measure the flux of radiated energy. And the term thermometer means that this instrument is capable of measuring the temperature of an object (with which it is in contact), but it is, practically, insensitive to the influence of the environment. So, four, closely related objects (gauge block and three thermometers) are in continuous interaction with a Dewar system, which transfers excessive energy and momentum to the temperature conditioning system and to the building, respectively.

In the case of our experiments demonstrating the invalidity of the superposition principle, there are already five material objects, which are located inside the Dewar, and three objects that transfer or store the excessive energy and momentum. Meanwhile, from theoretical physics it is well known that even for three rigid bodies, interacting gravitationally in accordance with the Newton's law, the general solution cannot be obtained in the form of closed algebraic expressions or integrals (H. Poincaré and H. Bruns, 1887). As the motion of three rigid masses is generally non-repeating (for the exception of a few special cases), S. Hawking in [7] summarizes this situation in theory in the form," We cannot even solve exactly for the motion of three bodies in Newton's theory of gravity, and the difficulty increases with the number of bodies and the complexity of the theory." Thus, even the thermal hysteresis effect cannot be adequately described in the language of mathematics. Consequently, it "makes absolutely no sense" (using the terminology of R. Penrouse in [33]) to speak about the adequate mathematical description of the thermal evolution process or of other Natural processes, as the EM interaction gives some contribution to any process on the Earth, and the thermal hysteresis effect is simply a thermal evolution process, which is recorded only at two different spatial locations of a material object. The conclusion is evident: the experimental studies of the thermal evolution process are far beyond the possibilities of description of theoretical physics.

Indeed, it is difficult even to imagine that there is a person nowadays, who has some relation to physics and still believe that mathematics can be applied for the description of the Poynting vector (which is produced by the EM field that is radiated by different parts of a material object in response to the external flux of energy), when the time delay, the spectrum and the flux of radiation from different parts of the surface of the object, as shown experimentally, are defined by the pre-history of the process and by the existing environmental conditions. When one had to take into consideration that the relation between the external and the internal EM fields cannot be found even for simple models of dielectric materials with absorption [24], and when for several decades, the experiments in the field of heat and mass transfer are giving indications that Fourier theory is in agreement with the measurements only in a steady-state phase, but not in the transient period of a thermal process.

From the considerations presented above, it follows that I. Newton, intending to obtain closed form or explicit 
mathematical solutions in his theory, had to use quite simplified approximation, which is based on:

1) the interaction between only two isolated bodies that is characterized by the concept of force, which is represented by the Third Law of Newton;

2) the extension of the two-body interaction to the multi-body case by the use of a simple mathematical procedure of summation of the forces to find the net force, which is postulated by the Second Law of Newton; this summing procedure is in dramatic contradictions with all natural processes, as the superposition principle is not valid in Nature;

3 ) the description of the effect of the resulting interaction (or the net force) against a fixed background, i.e., against the homogeneous time and the homogeneous space, which are postulated by the First Law of Newton.

It was Ernst Mach, who in 1883 publishing the famous book "The Science of Mechanics", gave incisive criticism of Newton's theory of dynamics [8] and enunciated the principle that now bears his name: "inertia is not is not an intrinsic property of matter or space, but depends on the existence of all matter in the Universe," [34] (pp. 443-445). Kleppner and Kolenkow emphasize in [34] that E. Mach pointed out that the fundamental weakness in Newtonian mechanics centers on Newton's concepts of space and time.

In his "Principia" [1] Newton writes, "Time, space, place, and motion, being words well known to everybody, I do not define. Yet it is to be remarked, that the vulgar conceive these quantities only in their relation to sensible objects. And hence certain prejudices with respect to them have arisen, to remove which it will be convenient to distinguish them into absolute and relative, true and apparent, mathematical and common, respectively." And after that Newton presents the definitions:

"Absolute, true and mathematical time, of itself, and by its own nature, flows uniformly on, without regard to anything external. It is also called duration.

Relative, apparent, and common time, is some sensible and external measure of absolute time (duration), estimated by the motions of bodies, whether accurate or inequable, and is commonly employed in place of true time; as an hour, a day, a month, a year."

And somewhat further Newton maintains: "It may be that there is no equable motion, by which time can accurately be measured. All motions can be accelerated and retarded. But the flow of absolute time cannot be changed. Duration, or the persistent existence of things, is always the same."

Mach comments in [8] (p.223) that "it would appear as though Newton in the remarks cited here still stood under the influence of medieval philosophy, as though he had grown unfaithful to his resolve to investigate only actual facts." Mach writes that, "views similar to those concerning time, are developed by Newton with respect to space and motion." Indeed, Newton in [1] maintains:

"Absolute space, in its own nature and without regard to anything external, always remains similar and immovable."

"Relative space is some movable dimension or measure of absolute space, which our senses determine by its position with respect to other bodies, and which is commonly taken for immovable space..."
And further Newton claims, "thus we use, in common affairs, instead of absolute places and motions relative ones; and that without any inconvenience. But in physical disquisitions, we should abstract from senses. For it may be no body really at rest, to which the places and motions of others can be referred..."

From the last part of this quotation it follows that as the material object cannot be found at zero temperature, and at the state of complete rest, so, the Newtonian space has no material counterpart, and thus, it is purely imaginary concept. Exactly the same conclusion has demonstrated Leibniz in paragraphs 4-7 of his third letter [4c], when using his Principle of Sufficient Reason.

Mach, criticizing Newton's absolute time, writes in [8] that "time appears to be some particular and independent thing", only if "the things we resort to for comparison" and for reference "play a wholly collateral part" in our mind. Further Mach emphasizes, "But we must not forget that all things in the world are connected to one another and depend on one another, and that we ourselves and all our thoughts are also a part of nature. It is utterly beyond our power to measure the changes of things by time. Quite on the contrary, time is an abstraction, at which we arrive by means of the changes of things; made because we are not restricted to any definite measure, all being interconnected."

"A motion may, with respect to another motion, be uniform. But the question whether a motion is in itself uniform, is senseless. With just as little justice, also, may we speak of an "absolute time" - of a time independent of change. This absolute time can be measured by comparison with no motion; and no one is justified in saying that he knows aught about it. It is an idle metaphysical conception."

In [34] Kleppner \& Kolenkow write (p. 444): "Mach goes on to point out that since time is necessarily measured by the repetitive motion of some physical system, for instance the pendulum of a clock or the revolution of the earth about the sun, then the properties of time must be connected with the laws which describe the motions of physical systems. Simply put, Newton's idea of time without clocks is metaphysical; to understand the properties of time we must observe the properties of clocks."

The observations of G.W. Leibniz, E. Mach and D.

Kleppner, presented above, are of primary importance for the formulation of the concept of time in relational World that is following below.

Mach's revolutionary ideas and his insistence that physical concepts be defined in terms of observables had no tangible immediate effect at that time, as all the theorists were raised on the reining theories based on Newtonian paradigm. Only forty years later, a shocking statement in the field of theoretical physics was made by Niels Bohr, who claimed that space and time description is not possible. The confirmation of this N. Bohr's position, which was in obvious contradiction with all principles of theoretical physics existing at that time, can be found in the very emotional letter of 1926 by Erwin Schrödinger to W. Wien: "Bohr's standpoint, that a space-time description is impossible, I reject a limine. Physics does not consist only of atomic research, science does not consist only of physics, and life does not consist only of science. The aim of atomic research is to fit our empirical knowledge concerning it into our other thinking. All of this other thinking, so far as it concerns the 
outer world, is active in space and time. If it cannot be fitted into space and time, then it fails in its whole aim and one does not know what purpose it really serves."

Quite soon, at the end of 1930, when many theorists were still recovering from Bohr's irritating observation, the new devastating blow came from the field of mathematics. Kurt Gödel made a public presentation of his first incompleteness theorem, which shows that "Any effectively generated, formal theory capable of expressing elementary arithmetic cannot be both consistent and complete." In other words this theorem states that any consistent formal system that includes enough of the theory of the natural numbers is incomplete: there are true statements (expressible in its language) that are unprovable by the system. And in 1931 the first incompleteness theorem was presented in his paper [6] "On Formally Undecidable Propositions in Principia Mathematica and Related Systems", where Gödel demonstrated the incompleteness of the theory of Principia Mathematica, a particular theory of arithmetic; but a parallel demonstration could be given for any effective theory of a certain expressiveness. It means that the basic claim of Newton's Principia that mathematics can be used as an adequate language of Natural Philosophy has been demonstrated to be false by purely mathematical methods. The important corollary of the incompleteness theorem is the existence of the infinitely many statements in the language of an incomplete theory that share the property of being true but unprovable. The parallel assertion in physics means that there is infinite number of experiments that falsify Newton's Mechanics.

It should be noted here that the great mathematician Carl Friedrich Gauss, whose contributions to mathematics and to theoretical physics are unprecedented, was actually predicting the existence of the famous Gödel's theorems. One of the C. F. Gauss's quotations reads, "Mathematics is the queen of the sciences and arithmetic (number theory) is the queen of mathematics." As soon as we realize that Gauss was meaning the superior generality of the number theory in comparison with the other fields of mathematics, we are coming to the first Gödel's theorem in the Kleene's formulation: "For any consistent, effectively generated formal theory that proves certain basic arithmetic truths, there is an arithmetical statement that is true, but not provable in the theory."

On the other hand, Gauss clearly realized the fundamental restrictions of mathematics, which examples can be picked up from his following quotations:

"Mathematics is concerned only with the enumeration and comparison of relations."

"There are problems to whose solution I would attach an infinitely greater importance than to those of mathematics, for example touching ethics, or our relation to God, or concerning our destiny and our future; but their solution lies wholly beyond us and completely outside the province of science."

"We must admit with humility that, while number is purely a product of our minds, space has a reality outside our minds, so that we cannot completely prescribe its properties a priori." (Letter to Bessel, 1830).

From the first quotation we learn that, as "mathematics is only concerned with the enumeration and comparison of relations", so most probably, it is incapable of any adequate description of complicated biological processes in living objects, and "the problem of our future", in accordance with the second quotation is declared to be, "completely outside the province of science." And by the third quotation, C. F. Gauss expresses serious doubts in the validity of the Newtonian concept of space and, consequently, of the whole Newtonian approach. In the concept of space, C. F. Gauss is approaching very close to the general philosophical ideas of G. W. Leibniz, who associated the "reality outside our minds" with matter, and who considered that, "Space denotes an Order of Things which exist at the same time, considered as existing together; without enquiring into their Manner of Existing. And when many Things are seen together, one perceives That Order of Things among themselves," [4c] (§4). So, in accordance with G.W. Leibniz, the space is that order among the material objects coexisting at the same time that is perceived by human beings, and, "Space is nothing else, but That Order or Relation; and is nothing at all without Bodies," (\$5 in [4c]). When sharing that type of views, it looks impossible for C. F. Gauss that a set of numbers, which is the product of our mind, can be used for a priory characterization of the properties of space, which "has a reality outside our minds".

Very important ideas and considerations dealing with the description of Nature by theoretical physics were expressed in public by A. Einstein in the last period of his scientific activity. First, as it follows from the "Intellectual Autobiography" of R. Carnap [11], Einstein was not satisfied with a concept of time existing in the theories of that epoch, including the concepts following from his relativity theories. Not finding an adequate response of his colleagues, A. Einstein had some discussions with one of the most famous representative of mathematical logics of that time Rudolf Carnap, who recalled [11]:

"Once Einstein said that the problem of the Now worried him seriously. He explained that the experience of the Now means something special for man, something essentially different from the past and future, but this important difference does not and cannot occur within physics. That this experience cannot be grasped by science seemed to him a matter of painful but inevitable resignation." But the famous mathematician couldn't see any problem, and in "Intellectual Autobiography" it is written, "I remarked that all that occurs objectively can be described in science; on one hand, the temporal sequence of events is described in physics; and, on the other hand, the peculiarities of man's experiences with respect to time, including his different attitude towards past, present, and future, can be described and explained in psychology". But A. Einstein did not share that point of view, as R. Carnap had to write [11]: "But Einstein thought that these scientific descriptions cannot possibly satisfy our human needs; there is something essential about Now which is just outside the realm of science."

From the first part of Einstein's statement follows that an adequate description of the Now, i.e., the present instant in the Universe or in natural process, "does not and cannot occur within physics" and that is the "matter of painful but inevitable resignation" for him. As according to Einstein the "scientific descriptions cannot satisfy our human needs", in his opinion, there is clearly a deep crisis in theoretical physics. Similar position of Einstein is expressed in his letter to Schrödinger of 22.12.1950, where he writes: "It is quite hard to accept that we still are in the stage of babies in their 
diapers, and it is not surprising that the fellows are unwilling to admit this (even to themselves)." As "there is something essential about Now which is just outside the realm of science," this means for Einstein that the concept of time, which existed in theoretical physics of that period, is not satisfactory.

First, it is worth noting here that there is a common point between the A. Einstein position and our experiments in the understanding of the Now, or the present moment in the evolution process: it appears that the present moment of the evolution process cannot be described adequately by physics in principle. As it follows from the experiments, the reason for the thermal evolution process is the flux of the net Poynting vector through the surface of a particular artifact. But in order to determine the net Poynting vector at the surface of a particular artifact at some specified moment, it would be necessary to find the radiated field by each "important" material counterpart at all preceding moments, in order to take into account the time of energy propagation to the specified artifact and the possible re-emission processes among the material counterparts. Clearly, this is not possible to achieve theoretically, as three vector material equations (or constitutive relations), which are making the Maxwell system of equations complete, cannot be described by electrodynamics [36], or by any other part of theoretical physics. The radiated field can be measured (with some uncertainty) experimentally. But in the thermal evolution process, the experimentally confirmed irreversible time corresponds to the system, comprising the artifact with several attached thermometers and the external field; so, the irreversible time means in this case that the original evolution process of the artifact is distorted for ever by the attachment of the thermometers to a macroscopic object and their interaction with the EM field even for a relatively short period of time. The conclusion is dramatic: neither theory, nor experiment can describe the present moment in the natural, evolution process. Every measurement or observation does produce an irreversible perturbation of the original, natural process.

The reason for that Einstein's extraordinary position can be picked up from his well-known quotations:

"No amount of experimentation can ever prove me right; a single experiment can prove me wrong."

"As far as the laws of mathematics refer to reality, they are not certain; and as far as they are certain, they do not refer to reality."

Here, on one hand, the first quotation signifies that Einstein realizes that, in accordance with Gödel's theorem, his relativity theories are only partial theories and are not the general ones, and, consequently, there is infinite number of experiments that are able to falsify his theories. On the other hand, it means the acceptance and the application to theoretical physics the philosophical ideas of Karl Popper: no finite number of experiments can prove that a theory is correct, but only one experiment, performed under new conditions, can falsify (refute) the theory under test. But the ideas of K. Popper correspond only to the relational material world of G.W. Leibniz and E. Mach, where, according to Leibniz ( $\$ 36-37$ in [4]), even the "infinite train of detailed facts" does not contain the "sufficient reason or the ultimate reason" for a contingent fact (or for the result of a particular observation by a human being);. In the relational world, according to Leibniz, "every simple Substance is by its nature a concentration, and a living mirror of the whole Universe, according to its Point of view," ( $\$ 87$ in [4a]). So, the "ultimate reason" for any contingent fact dealing with any part of material world is "the whole Universe"- the "necessary and supreme substance", which is "unique and universal" and "contains as much reality as possible," $(\$ \$ 38-40$ in [5]).

From the concept of relational world immediately becomes clear the meaning of the second of the Einstein's quotations. Even if we assume that for the system of many interacting bodies, which are continuously changing the properties in the process of this interaction, and if we were able to write the corresponding equations, still the tools of mathematics would not give any sequence of states of that system in accordance with the H. Poincaré results of 1887. For such type of the experimental situation, Einstein writes that "the laws of mathematics are not certain." On the contrary, if we consider a two rigid-body system, where the predictions of mathematics are certain, it "makes absolutely no sense" to apply this approximation for the description of natural processes, where the principle of superposition is not valid. For this type of situation Einstein claims, "as far as "the laws of mathematics" are certain, they do not refer to reality."

Similar, but more precise and unambiguous statement belongs to the famous contemporary mathematician and theoretical physicist Roger Penrouse, who defines that any general theory, which is of profound mathematical beauty, "makes absolutely no sense", though it can agree well with a large number of experiments [33].

The fundamental statements of Einstein that "scientific descriptions cannot possibly satisfy our human needs; there is something essential about Now which is just outside the realm of science," are perceived by L. Smolin in his recently published book in the following way:

"There can be no mathematical equation that perfectly captures every aspect of the world, because one property of the real world, not shared by any mathematical equation, is that it is always some moment.

Darwinian evolutionary biology is the prototype, because at its heart is the realization that natural processes can lead to the creation of genuinely novel structures," ( $p$. XVI in [2]).

"The fact that there is an arrow of time means that every moment is unique. The universe is different at different moments of time; these differences show in the properties of galaxies or in the relative abundances of the elements," (p. 213 in [2]).

First, it is clear that L. Smolin shares, in principle, the A. Einstein's views on the limitations of mathematical descriptions, but his position is, probably, much closer to that of R. Penrose. In Smolin book we find:

"One of the most important lessons is that Nature cannot be captured in any single mathematical system. The universe simply happens. It is unique. It happens once, as does each event - each unique event - that nature comprises."

"Physics can no longer be understood as the search for a precisely identical mathematical description of any process in the Universe. That dream must be seen as a metaphysical fantasy, which is now blocking the path to further progress. 
Mathematics will continue to be a handmaiden to science, but it can no longer be the Queen," [2] (pp. 240-251).

The last quote is evidently a natural development of the famous N. Bohr's idea that "Physics is to be regarded not so much as the study of something a priory given, but rather as the development of methods of ordering and surveying human experience. In this respect our task must be to account for such experience in a manner independent of individual subjective judgment and therefore objective in the sense that it can be unambiguously communicated in ordinary human language."

Second, the interpretation of the concept of the Now, or of the present moment in any process of the Universe, is of paramount importance for Einstein, as it plays a special role in his theories. As pointed out earlier in this paper, arrows of time (or irreversible processes) are typical for Astronomy, Biology, Zoology, Geology and some parts of experimental physics. Smolin adds one more: "There is another — less apparent, but nonetheless a major clue. Light moves from the past into the future. Hence, the light that reaches our eyes gives us a view of the world in the past, not the future. This is called the electromagnetic arrow of time, " [2] (p. 205). And each experiment, which is dealing with the observation of the arrow of time (in accordance with the second Smolin quote), means that every moment in the process is unique, so that all these processes are irreversible in time, and each of these types of experiments can be used for the falsification of any theory in which the "laws of physics are time-reversible". One of these theories "is Newtonian mechanics, another is general relativity, still another is quantum mechanics," [2] (p. 52).

Third, from Smolin quotations we find that the "evolutionary biology is the prototype" for natural sciences, "because at its heart is the realization that natural processes can lead to the creation of genuinely novel structures". This is in agreement with our studies of the thermal evolution process, which present a direct experimental support to one of the pillars of the Ancient Buddhism philosophy that "properties and structure of any material object are defined by the processes that occur inside and outside that object," [19] (p.34). As specially emphasized there, "the basic result of the study is the experimental demonstration that thermal evolution means the appearance of new properties in the object, which were not present in the parts of it, before the open system absorbed the momentum and energy of the external EM field." This is a direct experimental confirmation in the particular case of the electromagnetic field of a more general principle of driven self-organization, which is formulated in [2] (p. 219) as, "Flows of energy through open systems tend to drive them to states of higher organization." The results of our present experiments and conclusions are in deep agreement with the Ancient Indian (Jain) philosophy. "As per Jain dialectics, the truth and reality are perceived differently from different points of view; so, no single point of view can represent the complete truth. The fundamental Jain doctrine (Anekantavada) states that:

1) all entities have infinite numbers of qualities and modes of existence;

2) these entities cannot be completely perceived in all their aspects and manifestations by human beings, as a result of the inherent, intrinsic limitations of a human being," (p.39 in [16]).

To realize what impact and advancements presented A. Einstein's ideas about the relational world in physics, it is sufficient to compare some of the observations of $\mathrm{R}$. Feynman made during his lifetime. In the course of the University physics (1964), which was published almost 10 years after Einstein's death, Feynman writes that, "all electrons are the same, all protons are the same, all positive pions are the same; and so on..." [3a]. And on the next page in [3a] we read: "Apparently it is true that the fundamental physical laws, on a microscopic and fundamental level, are completely reversible in time". But several years later, after some crucial change of his points of views, R. Feynman was bitterly musing at one his interviews [2] (p. XXVI): "The only field which has not admitted any evolutionary question is physics. Here are the laws, we say... but how did they get that way, in time? So, it might turn out that they are not same all the time and that there is a historical, evolutionary question".

The person who shared and struggled for the spread of the Einstein ideas was Wolfgang Pauli. In his letter to Niels Bohr of 1955 [35], W. Pauli writes:

"To me it seems quite adequate to call the conceptual description of nature in classical physics, which Einstein wants to keep so emphatically, the ideal of the detached observer. In drastic words the spectator must, according to this ideal, appear in a fully discrete manner as a hidden spectator. He can never appear as an actor. Nature is hereby left alone in its predetermined course of events, without regard to the manner in which the phenomena are observed.

Since we can regard the measuring instruments as a kind of extension of the observer's sensory organs, I see the unpredictable change of the state through the individual observation - in spite of the objective character of every observation under the same circumstances - as a rejection of the idea of the detachment of the observer from the course of physical events outside of himself."

From the first passage we learn that A. Einstein wants to keep so emphatically the conceptual description of nature under the approximation of an ideal detached observer. In drastic words, under this ideal case, the spectator must appear in a fully discrete manner (as a hidden spectator), but he should never appear as an actor. The Nature should be left alone in its predetermined course of events.

The part of the Pauli quotation, "Since we can regard the measuring instruments as a kind of extension of the observer's sensory organs, I see the unpredictable change of the state through the individual observation", could be included as an explanation of the experimental results in [19] and in the present paper. Indeed, it follows from our experimental studies that as the thermal evolution process is irreversible, the installation of the thermometer on the surface of the gauge block spoils forever the original evolution process in the block by changing its pre-history. When passing the current through the thermometer, we produce much larger perturbation of its pre-history, and of its original properties, and of its structure. The result of the measurements refers only to the total system (the gauge block, thermometers and the external EM field), and the information about the original state of the block is lost forever. The process is irreversible in time, so that the state of 
the block before the measurement procedure cannot be restored under any experimental conditions. Consequently, the measurements with "the same block" under some smaller level of its perturbation cannot be realized in principle. So, this experimental situation illustrates Pauli basic statement about the inevitable "unpredictable change of the state through the individual observation," and emphasizes the importance of Einstein appeal that "the Nature should be left alone in its predetermined course of events."

In his other letter of that period [37], in 1951 Pauli writes: "That which is physically unique cannot be separated from the observer anymore - and therefore falls through the net of physics. The individual case is occasio and not causa. I am inclined to see in this "occasio" - which includes the observer and his choice of the experimental setup and procedure - a "revenue" of the "anima mundi" (of course in "changed shape") that was pushed aside in the 17th century. La donna é mobile - also the anima mundi and the occasio."

One can find that this short passage contains many fundamentals of the relational Leibniz's philosophy, as soon as we change the Latin terms "occasio" and "causa" by the English versions (the "contingent fact" and "sufficient reason"), which are used in the translations of Leibniz documents. And then we find that for Pauli, any individual case of observation is only a contingent fact, which includes the observer, his choice of the experimental set-up and of the measurement procedure, but which does not contain the sufficient reason for that observation. For Pauli each contingent fact is a "revenue" of the "anima mundi", or in other words, the result of each observation inevitably reflects the existence of the whole surrounding World (which is one of the basic principles of Mach's perception of the material world). When using Leibniz's terminology, in this case it is sufficient to say that each material object, which is used in the measurement procedure, "is by its nature a concentration and a living mirror of the whole Universe, according to its Point of view," or its "perspective on the Universe". The term "anima mundi" of the 17th century is not quite precise from the point of view of Leibniz's terminology, as it follows from the paragraphs 29-37 in [4b]. (It is clear that W. Pauli was not aware of the existence of the W.G. Leibniz - S. Clarke correspondence, as the vast majority of his contemporary theoretical physicists.)

The presented excerpt contains another Pauli assertion of paramount importance. According to Pauli, every material object under the study or observation, "which is physically unique" and which" cannot be separated from the observer" and from the whole external World, "falls through the net of physics." It means that theoretical physics is incapable of the adequate description of any measurement process, as the measurement instrument (which cannot be separated from the object under measurement), the material external World (which forms the spatial reference in the process, but simultaneously affects the properties and the structure of the object), and the clock (which is used as a reference in time, but also participates in the interaction process with the measured object) - are all beyond the scope of description of theoretical physics as a result of its internal restrictions. So, the Pauli assertion, "That which is physically unique cannot be separated from the observer anymore - and therefore falls through the net of physics," is equivalent in many respects to
Einstein's statement that "scientific descriptions cannot satisfy our human needs."

Before formulating the concept of time in a relational World, it is would be useful to discuss some other results of our studies of the thermal evolution process.

3. The very existence of the thermal surface energy, when in response to external EM radiation fluxes of energy and momentum do propagate large distances inside a material object [20], has demonstrated experimentally that the isolated system approximation of theoretical physics (often called as doing physics in a box) is absolutely incompatible with the present day development of the experimental science.

4. It is shown experimentally in [17] that the fluxes of energy and momentum (described by the Poynting vector) through the boundary of an artifact are the driving sources for the arising thermal evolution process, when the properties and the structure of the artifact are continuously changing in response to the external factors [19].

5. The common EM field, which is created by the huge number of material objects in response to the radiation of the stars, carries the information about the hysteresis effects in each part of every material object and thus presents the history of the evolution process of the material world up to the particular moment of observation at a specified, particular point of the Universe [19]. So, the field describes the result of the evolution process of the whole material World at a particular point of a specified artifact, and simultaneously this field is the source of the future changes of the properties and of the structure of this particular artifact that are permitted by the hysteresis effects in the artifact and by its future external conditions. In this way the "harmony" is established, when according to G. W. Leibniz (1715), "every simple substance is by its nature a concentration and a living mirror of the whole Universe, according to its Point of view," or its "perspective on the Universe." Clearly, this common field is representing material process, and, in principle, it cannot be described in terms of mathematics. In Maxwell's equations $[25,36]$, in the general case, the EM field and polarization should be perceived as processes, and the mathematical description of these physical quantities is only possible under extremely primitive theoretical approximations.

6. The experimental studies performed in [19] have shown that the violation of the symmetry in space (illustrated by Figs. 5a-5c, there) and breaking of the law of the momentum conservation of the system do occur in the experiment strictly in parallel. Indeed, the reflection of the energy flux from the gauging surface results in the surface energy build-up and the temperature asymmetry of the system [21], the same process can be treated as the reflection of the field-particle momentum [20] from the same surface that results in the symmetry braking of the momentum, as the compensating external impulse is applied to the block through the block supporting mechanism. In a similar way, when the record of the hysteresis loop in Figs. 6a-6c (presented here) is obtained, it means that the energy of the system is not conserved, as the area of the hysteresis curve is describing the amount of thermal energy that is removed by the air conditioning system from the material objects during one modulation cycle; on the other hand, the record of the hysteresis loop means the realization of the cyclic process that is irreversible in time, as it is shown in [17], in accordance with the Plank's formulation of the second law of 
thermodynamics. So, the observation of the irreversible time (or time asymmetry) in the thermal evolution process is equivalent to the observation of breaking of the law of conservation of energy. Taking into account the experimental plots in Figs. 10 and 11 in [19], we conclude that braking of symmetries in the thermal system occurs within 1 minute in a tungsten carbide block after the application of the modulation signal, while the maximum variation of the temperature within the source of modulation (PRT) never exceeds $60 \mathrm{mK}$ relative to the block temperature! So, under these experimental conditions, the standard approximation of an isolated system is becoming invalid in 1 minute, so that the laws of conservation of the energy and of the momentum, as well as E. Noether theorem [38], are also loosing validity in one minute, while the tungsten carbide block can exist hundreds of years under these conditions.

Before defining the concept of time, which corresponds to the relational world and which is in agreement with the existing Universe, it is necessary to realize that the concepts of time, which existed at the time of A. Einstein, were not satisfying him, or simply "wrong" (when using Einstein's terminology). To demonstrate this assertion it is sufficient to use an extremely powerful tool, which Einstein has introduced into physics and which is known as Popper-Einstein method. This method is capable to demonstrate immediately that a scientific theory under examination should be considered as a "falsified" one; according to this method, a single experiment, which is in contradiction with the predictions of the tested theory, is sufficient to falsify this theory. For example, the astronomical observations of Arthur Eddington (mentioned above) or the irregularities of the Earth's rotation [28] are sufficient to falsify the whole Newtonian physics, including, naturally, his concept of time, whose properties are following from the First Law of Newton. The concepts of time by A. Einstein (following from the General and Special Relativity Theories) are falsified by the numerous experiments in [39] (Figs.11, 12 ), which clearly indicate that the frequencies of the primary frequency standards, as well of the time-keeping devices (clocks), are never coincident [39,40], even after applying the corrections on the gravitational and relativistic effects. Besides that, the results of the future deviations of the corresponding time scales cannot be predicted using the tools of mathematics, even in cases with absolutely huge amounts of the previous experimental comparisons. (The situation is quite similar to the mathematical analysis of the Earth's rotation.) The Einstein Relativity theories (as well as the other scientific theories) are falsified by the experiments, in which the hysteresis effects and velocity dispersion are observed in material objects, in which the perturbation of the investigated system and the detection of its changing states are performed by using EM fields, only. Also, the experiments that are giving support to the General Relativity Theory falsify the Special Relativity Theory, and vice versa. It means that these Relativity theories are based on different assumptions, and the corresponding Einstein concepts of time are not general and are presenting a good first-order approximation only under the conditions of the particular theoretical model.

To understand better the principles of Leibniz's views on the relational world, the comparison of a short passage from paper [19] (which was written before studying the original
Leibniz's documents) with some parts of Leibniz's correspondence [4] will be performed. As some intermediate summary of the results of the paper on page 80 in [19] it is written: "As in natural selection in biology, in our experiments there is always infinite number of external parameters. The Poynting vector of the external field is the result of the interaction between the numerous material objects during the whole preceding time epoch, including the instant of the last measurement point in the series. The EM field, re-emitted by the each object, depends on its own pre-history, simply as a consequence of the existence of the surface energy and the accompanying hysteresis effect. The evolution process in the studied artifact presents the synthesis process, occurring under the influence of infinite number of internal and external influence factors. And the basic result of this study is the experimental demonstration that thermal evolution process, or thermal synthesis, means the appearance of the new properties in the object, which were not present in the parts of it, before the open system absorbed the momentum and energy of the external EM field".

The experimental plots of Figs. 6a and 6b in [19] are sufficient to make a conclusion that the Poynting vector of the EM field is the source of the hysteresis effect and of the thermal evolution process. So, if we call for a while in this example the fluxes of energy and momentum of the common field (that is created by all material bodies in the vicinity of a specified material object) by the Leibniz's term "the Efficient Cause", which he uses in $\S 124$ in [4a], then we can trace how physical interactions between material objects can manifest themselves in the Leibniz's relational World. According to G. W. Leibniz ( $\$ 87$ in [4a]), "every simple Substance is by its nature a concentration, and a living mirror of the whole Universe, according to its Point of view," and this, "is one of the most beautiful and most undeniable Proofs of the existence of the Efficient Cause; since none but the Efficient Cause, can produce such a Harmony of things." One of the basic principles of the relational world is outlined in $\$ 10$ in [5], where Leibniz writes, "I assume also as admitted that every created being is subject to change, and further that this change is continuous in each."

And in the $\$ 91$ in [4a], he clarifies, "The Nature of every simple Substance being such, that its following State is a consequence of the preceding one; here now is the cause of the Harmony found out. For the Efficient Cause needs only to make a simple Substance become once and from the beginning, a representation of the Universe, according to its Point of view; Since from thence alone it follows, that it will be so perpetually; and that all simple Substances will always have a Harmony among themselves, because they always represent the same Universe." 
After these Leibniz's quotations, it is clear that the concepts of time and of space, which are formulated in accordance with the Newtonian paradigm, are becoming unacceptable. The basic features of these concepts were formulated by G. W. Leibniz in [4c]. Leibniz writes in $\S 4$ of [4c]: "I have said more than once, that I hold Space to be something merely relative, as Time is; that I hold it to be an Order of Coexistences, as Time is an Order of Successions. For Space denotes an Order of Things which exist at the same time, considered as existing together; without enquiring into their Manner of Existing. And when many Things are seen together, one perceives That Order of Things among themselves." And further in $\S 6$ of [4c] Leibniz writes, "Instants, considered without the Things, are nothing at all; and that instants consist only in the successive Order of Things or successions of States."

So, it is clearly maintained that space and time express different, complementary types of relations among the material objects and that these concepts do exist only within the material World. In [4a] G. W. Leibniz repeats that, "Time, considered without the things, being an impossible fiction" (§58); "Space out of the World must be considered imaginary," (\$33). The further development of this Leibniz's position we find in the $\S 105$ of [4a], where defending the thesis of his third letter that, "Time is an Order of Successions," he refutes the objection of Dr. Klarke, who considered "that Time cannot be an Order of successive Things, because the Quantity of Time may become greater or less, and yet the Order of Successions continue the same." In reply Leibniz writes [4a], "This is not so. For if the Time is greater, there will be More successive and like States interposed; and if it be less, there will be fewer." Thus, according to Leibniz, the concept of time, using the contemporary terminology, is associated with the material process, and the measure of time is related to the number of the "successive and like States" of a material object in that reference process.

Of primary importance is the Leibniz's observation that the time is not homogeneous in the relational World. Indeed, the time is characterized by the "successive and like States interposed" in this material, reference process. These are only like or similar states in this reference process, just because the identical states are simply impossible in the relational material World, in which (according to $\$ 10$ in [5]),"every created being is subject to change, and this change is continuous in each." And in this material World, the "Efficient Cause" "makes changes according to the principle of the best," ( $\$ 48$ in [5]); and "in simple substances the influence of one Monad upon another is only ideal, and it can have its effect only through the mediation of the "Efficient Cause"... Since one created Monad cannot have any physical influence upon the inner being of the other, it is only by this means that the one can be dependent upon the other." And the "definition" of the "Efficient Cause" we find in $\$ 124$ in [4a], "All the natural forces of Bodies, are subject to Mechanical Laws, which follow the Order of Efficient Causes."

Thus, the Leibniz's observations are acquiring deep physical meaning, when substituting the old term ("Efficient Cause") by a new one - energy flux of a common field. As the common field, which is created by all material bodies during the whole pre-history up to the present instant at the position of the selected material object, defines the processes, properties and the structure of this object, this common field realizes the "pre-established Harmony" in the Universe in the form of a continuous, irreversible evolution process, when material objects are gradually adjusting their structure and properties to the continuously changing external conditions.

As it follows from the above presented quotations from Mach's book [8] of 1883, his views on the time and space were very close to the ideas of Leibniz. And the following quotation of Mach is of primary importance for the formulation of the concept of time: "We must not forget that all things in the world are connected to one another and depend on one another, and that we ourselves and all our thoughts are also a part of nature... Time is an abstraction, at which we arrive by means of the changes of things; made because we are not restricted to any definite measure, all being interconnected," [8] (pp. 223-224). Using the present day notions, we can define time as a natural reference process. Indeed, according to Mach, to time "we arrive by means of the changes of things," and "all things in the world are connected to one another and depend on one another." And changes of things in the relational World, in which all things are interconnected, or each thing "is by its nature a concentration, and a living mirror of the whole Universe, according to its Perspective on it," in accordance with the results of this study is called a process. This concept of the process is naturally found to be in the strict agreement with the Leibniz's definition of time, when recalling Leibniz's quotations from his third and fifth letters "Time is an Order of Successions" or "an Order of successive and like States" of some process. Thus, we are to relate the Leibniz-Mach concept of time with the natural reference process. As shown experimentally in this paper, the process cannot be described by mathematics (i.e., by any single homogeneous time) and has infinite number of influence factors (representing all interacting material bodies), or using Mach's terminology the process is "not restricted to any definite measure," as "all things in the world are being interconnected." So, our experimental results confirm the fundamental Mach's statement that "With just as little justice may we speak of an "absolute time" - of a time independent of change. This absolute time can be measured by comparison with no motion; and no one is justified in saying that he knows aught about it. It is an idle metaphysical conception," [8] (p. 224).

After realizing that the concept of time in the relational material World is related to a natural process, all the missing elements of the procedure of the measurement in time can be borrowed from Leibniz's observations. According to Leibniz, time and space are complementary notions that are characterizing the material World. And in [4c] Leibniz writes, "I hold Space to be something merely relative, as Time is." And in $\S 4$ in [4c] we find, "Space denotes an Order of Things which exist at the same time, considered as existing together. And when many Things are seen together, one perceives That Order of Things among themselves." From this quote we find that the Order of Things is perceived at the place of observer, and the record of the Order is performed at same time, i.e., using the clock, which is located at the place of the observer. Naturally, this Order or Space depends on the relative position of the observer among the material objects and on readings of the clock, which is 
available at the point of observation. Absolutely symmetrically, to realize the process of measurement in time, we must have available, at least, two natural processes at the place of a recording instrument (or observer), when one of the processes is chosen as a reference and it is called time, and the sequence of consecutive states of the other process is recorded by the measuring instrument as a function of time at the spatial position of the recorder. As it follows from the presented experimental studies, the measurement procedure presents an irreversible process, and the result of the measurement pertains to both natural processes, to the nonlinear recorder (with its inevitable delay in time or hysteresis effect), and to material environment, with which the measurement system is constantly interacting.

And from the definition of the measurement process it follows that the reference process or time is always perturbed in the irreversible way by the inevitable interaction of the "clock" (i.e., the material object in which the reference process is realized) with the surrounding material objects in the Universe. And here, it is possible to discern two different channels of perturbation. The first channel is produced by the links of the measurement procedure, when the studied process produces the perturbation of reference process through the recorder (nonlinear device with the hysteresis effect), as some parts of the external fluxes of energy and momentum, which are associated with the studied process, do penetrate inside the recorder an then the EM energy is radiated from the surface of this material object, as it is demonstrated in the studies of the surface energy $[16,17]$. The second channel of the perturbation is through direct influence of the Poynting vector of the common field on the reference and on the studied processes, again as a consequence of inability to create an isolated system (due to existence of the surface energy). The relative contribution of these channels depends on the construction of the clock and on the properties of the comparison system, but always both of the compared processes are under the continuously changing perturbations. The difference in perturbations, which are acting on two clocks spatially separated, can be measured in a standard way for arbitrary time standards. It is described by the Allan variance [41]. Dealing with topics of this paper, the most interesting for us is the specific increase of the Allan variance as a function of the averaging time (after reaching the "flicker floor") that is observed for any type of frequency standards [39, 40]. It is the other direct experimental manifestation of the Leibniz's "Law" that every material object "is a concentration and a living mirror of the whole Universe, according to its Point of view" and of the principle of Identity of Indiscernibles (both of which are experimentally confirmed by the present studies). Indeed, for two different positions of the two clocks (or frequency standards) the Poynting vectors of the common field cannot be identical (due to the principle of the Identity of Indiscernibles), and, consequently, the clocks, which are "the living mirrors of the whole Universe, according to their points of view" will inevitably produce different rates (frequencies) and different time scales. So, the time in the relational World cannot be homogeneous, as the procedure of generation of the states in the reference process is always perturbed in an irreversible way by the external sources EM radiation, as demonstrated experimentally in this study. In this respect, the inventor of the hydrogen maser, Daniel
Kleppner, writes in his book, "Mach goes on to point out that since time is necessarily measured by the repetitive motion of some physical system, for instance the pendulum of a clock or the revolution of the earth about the sun, then the properties of the time must be connected with the laws which describe the motions of physical systems. Simply put, to understand the properties of time we must observe the properties of clocks," [34] (p. 444). And the properties of the clocks can be found, for example, from the description of the experiment of Hafele and Keating [42], which is often referred in literature as a "proof" of the Einstein Relativity Theories. In this experiment, four commercial caesium clocks, after comparison with the time scale of the US Naval Observatory, were used in the airplane flight experiment around the Earth: one flight was in the eastward direction, the other was in the westward direction. In the eastward trip, the ensemble of four clocks lost on average 59ns relative to the USNO standards, and one standard deviation for the readings of the flying clocks was 10ns. One can easily find that for four independent clocks the estimated uncertainty of the mean value in the ensemble is several tens of nanoseconds, which is quite comparable with the claimed mean value. But the evident result that was proved by that experiment is the fact that all the flying clocks indicated different values in the comparisons with USNO standards, and their rates has changed as a result of the flight (that is hysteresis effect has been observed), and these changes were specific for each clock [42] (see Figs. 1 and 2). No effects of this kind can be predicted by any of the Einstein Relativity Theories. It is simply fascinating that the some people believe that one experiment can prove even the combinations of two theories, which are based on totally different assumptions and models. Thus the flying clocks experiment has demonstrated that the time scale of each clock depends on a huge number of external factors, and not only on the gravitation or the clock motion relative to the standards stationary on the Earth. These aspects were emphasized by Louis Essen, the creator of the first primary caesium standard, who wrote critical comments on Hafele-Keating experiment:" One aspect of this subject which you have not dealt with is the accuracy and reliability of the experiments claimed to support the theory. The effects are on the border line of what can be measured." And L Essen continued," The authors tend to get the result required by the manipulation and selection of results. This result was published in Nature, so I submitted a criticism to them. In spite of the fact that I had more experience with atomic clocks than anyone else, my criticism was rejected."

As pointed out above, the Leibniz - Mach concept of time is completely different from the meaning of "time" in the Special Theory of Relativity (STR), for example. Here, it is necessary to realize that Einstein's STR is based on the Newtonian principles of description of Nature in combination with two postulates, which are in agreement with the $\mathrm{H}$. Poincaré criticism of the Galilean simultaneity for spatially separated events that was known since 1898 [43] (p. 655). The Einstein's postulates are formulated in terms of inertial reference systems [43], which are defined in agreement with the First Law of Newton that postulates the possibility of a body motion in straight line with constant velocity magnitude. But such motion can be assumed only for the imaginary Galilean (Descartes) reference frames and not for a material object that is moving relative to the stationary 
ensemble of material counterparts (i.e., space in accordance with Leibniz and Mach). In the latter case, the motion of the artifact with constant velocity is basically impossible (in accordance with the Lenz law) due to the presence of the thermal radiation from the optically discernible material counterparts, and also, due to the existence of the gravitational force between the object and environment. As the absolute Newtonian time is not compatible with the STR, the concept of velocity in STR (including the velocity of light) is becoming defined only after the introduction of the synchronization procedure [44]. The choice of the synchronization procedure results, naturally, in the corresponding consequences. Einstein "overcame" this problem by postulating that the synchronization of two spatially separated clocks could be realized by a short pulse of light without any perturbations of the clocks. So, it was assumed that the clocks were ideally isolated systems, which were free of external perturbations. And this is clearly again the approximation of I. Newton, who considered (in accordance with Newton's Third Law) that two interacting material object could form an isolated system and that the interaction between them was free from any environmental effects.

At last, when finding the final relations between the spatial coordinates and the time instants in two inertial frames (that are known as Lorentz transformations), Einstein supposed that space was homogeneous and isotropic, and time was homogeneous in both inertial systems [43] (p. 671). As shown in [43] (p. 672), when writing for both inertial reference systems the linear mathematic relations between the time intervals in both systems (which are the consequences of the assumed homogeneity and isotropy of space and time in this theory) and then taking into account the equivalence of two reference systems and that the speed of light in both inertial system is the same (i.e., Einstein postulate), one immediately receives the Lorentz transformation. This means that again, as in the case of Newton's Second Law of mechanics, the rules of mathematics were used to obtain the solution in a more general case when starting from the oversimplified case of the isolated system. Thus, STR was developed in accordance with the Newtonian paradigm, and consequently, in accordance with the first K. Gödel's theorem, STR is not a general theory, but, using the terminology of S. Hawkins [7], it is only a "partial" theory, which can be falsified by enormous number of experiments.

Next, dealing with the Einstein's choice of the method of synchronization of the clocks, one can come to the conclusion that Einstein's information about experimental techniques existing at that time was, probably, quite limited. Indeed, the best time standards of that period were the pendulum Riefler clocks, which about 20 years later were substituted by more sophisticated Shortt clocks [40]. Both of these unique devices by means of astronomical observations were calibrated to reproduce the mean Solar day [28] - the natural time standard, which was used by mankind for thousands of years. For both types of these time standards, the method of synchronization of two clocks by a single light pulse is found to be not possible. But even more dramatic is the fact that the very idea of Einstein to perform synchronization of two such clocks, which are spatially separated, looses any sense. This statement is becoming natural if one recalls that in relational
World, the properties of an object are dependent on the surrounding material environment, and the material environment is always changing when the object changes its position relative to the material reference. So, when we change the position of the pendulum clock, its rate is changed as a result of a huge number of interacting bodies, and the variation of the height of the clock relative to the centre of the Earth very often gives one of the major contributions. Thus, by the sequence of a large number of light pulses, which are specially generated at the location of one of the clocks, we can perform only the comparison of the rates of the clocks, which are not moving relative to the Earth, but the information about the time instants reproduced by the clocks is not available in this procedure. The corresponding situation was described in a popular Soviet text-book for Universities, which was written before 1980 by the author, who sincerely believed that Einstein General Theory of Relativity was not a partial, but general theory. The translation of the passage can be presented in the following way: "As in the presence of a gravitational field the rates of identical (similar) clocks located in different points of space are different, then the method of establishing simultaneity between the two spatially separated events and the method of the clocks synchronization in STR, are loosing sense. In the presence of the gravitational field, only the local time, which in each point of the space is established with the help of identical traveling clocks, has the meaning. In this case, we are to speak not about the synchronization of the spatially separated clocks, but only about the comparison of the rates of the clocks," [43] (p.701).

And the last remarks about the STR can be made on the basis of the present day experimental information. First, it deals with the STR assumption that the speed of light in all directions is the same. This assumption is only suitable for an empty space, but not for the material world. And the paper of B. Nodland and J. P. Ralston "Indication of Anisotropy in Electromagnetic Propagation over Cosmological Distances" [45], which is reporting experimental dependences on the direction and polarization of electromagnetic radiation propagating over cosmological distances and thus revealing a true anisotropy in the structure of our Universe, seems to be quite sufficient to falsify this assumption of STR.

Second, remark deals with the Einstein's assumption that the maximum value of the speed of light $c$ is equal to $3 \times 10^{8} \mathrm{~m} / \mathrm{s}$, as it is known from literature that "the theory of special relativity excludes faster-than-light speeds a priory," [44]. But in the experimental study of S. Chu and S. Wong [46] it is shown that a short laser pulse can propagate in an absorbing medium in a linear regime, when the saturation effects can be neglected, without a significant change of the shape of the pulse. The velocity of the propagation of the envelope of this laser pulse inside the medium can be described by the group velocity [47], which can be smoothly changed in the experiment by the variation of the absolute value of the frequency detuning of the pulse relative to the absorption line center. For small frequency detuning of the laser pulse (in the frequency band of the anomalous dispersion of the medium), the maximum of the laser pulse, which is leaving the sample, appeared at the time instant when the maximum of the original laser pulse had not even entered the sample [46]. The last statement of the paper in the measurable parameters, which in this case are the time delay 
and the thickness of the sample, corresponds to the assertion that the propagation of the pulse with the speed of light $c$ would result in the time delay of the pulse leaving the sample, while the measured value indicated to the advance of the pulse. And the delay time equal to zero corresponds in mathematical terms to the group velocity equal to infinity. To emphasize that the group velocity is not equal to energy propagation velocity, Chu and Wong specially write, "if the pulse propagated with the energy velocity, a pulse delay of $6.7 \mathrm{ps}$, rather than a pulse advance, would have been seen." When summarizing the results in [46], Chu \& Wong claimed: "In this work, the pulse velocity not only exceeds $c$, but is measured to go smoothly through \pm infinity, and, in some samples, to have a negative value as low as $-1 \times 10^{-8} \mathrm{~cm} / \mathrm{sec}$." In accordance with internet information, similar type of experiment was performed by Lijun Wang with colleagues in 2015 from the NEC Research Institute in Princeton. In this case, the laser pulse was passed through a relatively long absorption cell, which was filled with super-cooled caesium gas obtained as a result of optical laser pumping. As in previously described experiments of S. Chu and S. Wong, the time advance instead of the time delay for the corresponding laser pulse was observed. Lijun Wang maintains that, "our experiment does show that the generally held misconception that "nothing can travel faster than the speed of light" is wrong." At last, the other recent experimental study of the entangled electron spins by Hensen et al [48] "Experimental loophole-free violation of a Bell inequality..." also seems not to be in favor of the predictions of STR about $c$.

From the discussions presented above it follows that both the Special and the General Relativity Theories were developed in accordance with Newtonian paradigm and the additional Einstein postulates, which are only valid under quite specific experimental conditions, can be regarded as some additional axioms in the structure of these mathematical theories. So, in accordance with K. Gödel incompleteness theorem, STR and GTR should be regarded as incomplete theories, which can be falsified by enormous amount of experiments and which concepts cannot be extended beyond the experimentally determined regions of validity of these approximate, partial theories. For example, the Lorentz transformation and the concepts of time in STR and in GTR are falsified by experiments demonstrating the electromagnetic arrow of time; by experiments describing the invalidity of superposition principle in the interaction of the EM field with matter $[49,16]$; by experiments dealing with hysteresis effects in interactions between EM field with matter, as well as by hysteresis effects in constant magnetic and electric fields. So, when A. Einstein realized that during all his life he was not able to get rid of I. Newton approximations, which are based (according to Mach) on the medieval philosophical principles, and that "the experience of the Now means something special for man, something essentially different from the past and future, but this important difference does not and cannot occur within physics," he made a public statement that, "No amount of experimentation can ever prove me right." The only way out, Einstein saw in the "painful but inevitable resignation." When Einstein wrote in his letter to Schrödinger of 1950, "It is quite hard to accept that we still are in the stage of babies in their diapers," [50], this was the realization of a deep crisis in theoretical physics, about which R. Feynman was musing at one of his press-conferences [2] (p. 52): "The only field which has not admitted any evolutionary question is physics. Here are the laws, we say. But how did they get that way, in time? So, it might turn out that they are not same all the time and that there is a historical, evolutionary question." The meaning of this quote is dramatically different from what Feynman wrote in his University text-book in 1964. The earlier statement of R. Feynman in [3c] was, "the mathematical equations of the physical laws must be unchanged under a Lorentz transformation." It is also indicating to the deep problems in theoretical physics. First, the Lorentz transformation does not possess the necessary level of the generality, being in obvious contradiction with all the experiments demonstrating different types of arrows of time; in particular, with numerous experiments on thermal conductivity, in which the time is clearly irreversible. Second, the Lorentz transformation is falsified by the experimentally established fact of the velocity dispersion is present in the interaction between the EM field and the ensemble of atoms (see, for example, Fig.1 in 15]). Under these conditions, the foundation for the Feynman's statement can be easily revealed. If the mathematical equations of some partial theories are not changed under the Lorentz transformation, it simply means that all these theories were developed in accordance with the Newtonian paradigm, e. i., were based on the medieval approximations of I. Newton (in accordance with the views of E. Mach). The notions of time and space, which are following from the partial physical theories based on Newtonian paradigm, cannot meet the experience of the mankind (according A. Einstein) and cannot agree with the corresponding concepts of such Natural sciences as Biology, Zoology or Geology, for example. The notions of "time" in these partial theories are not in agreement with each other, and they can be regarded only as useful variables in the calculation procedures of a particular theory. These notions of "time" are unable to compete with the general concept of time in the Natural Philosophy of G. W. Leibniz and E. Mach. Here, it should be also noted that the notions of velocity and derivatives, which are purely mathematical concepts, are applicable in the relational World only in case of very slow processes. For the fast process, which is under investigation, the short-term stability of the reference clock may be already insufficient, so that the limit of the ratio of the variation of some quantity to the time interval, when the time interval is approaching zero, is automatically loosing sense. In this case, it is only possible to speak about a mean value of this ratio for long averaging times, but the concepts of derivatives and velocity have no meaning. In this respect, special importance acquires the remark by S. Chu and S. Wong in [46]," Although the delay as a function of laser frequency is a smooth, well behaved function, the pulse velocity goes through some rather counter-intuitive singularities."

This paper is an experimental one; the interpretation of the results and conclusions are based on the ideas of $\mathrm{G}$. W Leibniz, Ch. Darwin, C. F. Gauss, E. Mach, H. Poincaré, N. Bohr, K. Gödel, A. Einstein, W. Pauli, R. Penrouse, D. Kleppner, L. Smolin and others. The key features of the relational concept of time are the following ones. First, this concept is associated with the process, which is developing in a particular clock and which is always specific for particular position in the Universe (as demonstrated experimentally). 
Thus, the time scale is always local. Second, the relation between the two processes (or time scales) cannot be adequately described by any mathematical expression: the concept of process is far beyond the axiomatic approach of mathematics (as shown above). The concept of time in the relational World always is associated with the irreversible process, even in the case of purely electromagnetic type of interaction between the material objects. In this respect, is of special importance the observation of Wolfgang Pauli that, "which is physically unique falls through the net of physics. The individual case is occasio and not causa. I am inclined to see in this "occasio" - which includes the observer and his choice of the experimental setup and procedure - a "revenue" of the "anima mundi". La donna é mobile - also the anima mundi and the occasio." Here, the views of W. Pauli are in deep agreement with the fundamental principles of Gottfried Wilhelm Leibniz, who according to L. Smolin (p. XXVII in [2]) "has been called the smartest person who ever lived."

\section{ACKNOWLEDGMENT}

The author gratefully acknowledges the support of our studies at INMETRO by the National Research Council $(\mathrm{CNPq})$ of Brazil. Important technical contribution and assistance in our experimental studies by the INMETRO staff are highly appreciated. The author is grateful to the staff of the Physics Department of the Yeditepe University (Turkey) for useful discussions, the offered possibility to present the pioneering results at the International Conference "Chaos-2013" and for the support in the following publications.

\section{REFERENCES}

[1] I. Newton, "Mathematical Principles of Natural Philosophy." 1687; London: Dawson, 1969.

[2] L. Smolin, "Time reborn." Houghton Mufflin Harcourt, Boston - New York, 2013, p. XXI [2a]; p. XXIII [2b]; p. 53 [2c]; p. 52 [2d];

[3] R. P. Feynman, R. B. Leighton, and M. Sands, "The Feynman Lectures on Physics.” Addison-Wesley, 1-ed, V.1, Ch.52, 1964, [3a]; V.3, Ch.17, [3b]; V.3, Ch-52 [3c].

[4] H. G. Alexander, Ed, "The Leibniz-Clarke Correspondence." Manchester: Manchester University Press, 1956, Leibniz' s fifth letter [4a]; Leibniz' s fourth letter [4b]; Leibniz' s third letter [4c].

[5] G. W. Leibniz,. "La Monadologie." 1714, R. Latta, (trans.), Oxford: Clarendon Press, 1898; eBooks@ Adelaide, University of Adelaide, South Australia, 2014.

[6] K. Gödel, "On formally undecidable proporsitions of Principia Mathematica and related systems I." 1931, in Feferman, S. (Ed), "Kurt Gödel Collected works," V. 1, Oxford University Press, pp. 144-195, 1986.

[7]. S. Hawkins, "The illustrated a brief history of time." New-York: Bantam Books, 2008.

[8] E. Mach, "The Science of Mechanics." 1883, 4th ed, Open Court Publishing Co., Chicago - London, 1919.

[9] R. P. Feynman, "The Character of Physical Law." Cambridge, Mass: MIT Press, 1965.

[10] C. S. Peirce, "The Architecture of Theories" The Monist, 1:2, pp. 161-176, 1891.

[11] P. A. Schillp, (Ed.), "The Philosophy of Rudolf Carnap: Intellectual Autobiography.” La Salle, IL: Open Court, 1963, pp. 37-8.

[12]. A. Einstein, B. Podolsky and N. Rosen, "Can Quantum-Mechanical Description of Physical Reality Be Considered Complete?" Phys. Rev., V. 47, 1935, pp. 777-780.

[13] B. Greene, "The fabric of the cosmos: space, time and the texture of reality." New York: Alfred A. Knopf, 2006, p. 268.

[14] A. Titov, I. Malinovsky, "Nanometrology and high-precision temperature measurements under varying in time temperature conditions." Proc. SPIE, V. 5879, Recent Developments in Traceable Dimensional Metrology, J. E. Decker , Gwo-Sheng Peng, (Eds.), pp. 587902-1 - 587902-10, San Diego, CA, USA, 31 July-1 August, 2005.
[15] A. Titov, I. Malinovsky, "New techniques and advances in high-precision temperature measurements of material artefacts." Can. J. of Scientific and Industrial Research, V.2, No.2, pp. 59-81, 2011.

[16] A. Titov, I. Malinovsky, "Demonstration of the existence of thermal surface energy and its impacts." European Scientific Journal, V.9, No. 24, pp. 11-44, 2013.

[17] A. Titov, I. Malinovsky, "Experimental demonstration of time-irreversible, thermal evolution processes and some of its consequences." International Scientific Forum, ISF 2013, 12-14 December 2013, Tirana,

Albania, Proceedings ISF 2013: European Scientific Journal, February 2014 /SPECIAL/ edition, vol.3, pp.78-91, 2013.

[18] A. Titov, I. Malinovsky, "Experimental demonstration of time-irreversible, self-ordering evolution processes in macroscopic quantum systems." Chaotic Modeling and Simulation (CMSIM) V.1, pp. 79-93, 2014. [19] A. Titov, "First observations of thermal surface energy and of thermal evolution process." Journal of Science Frontier Global Research, (GJSFR), V. 15, Issue 3, Version 1.0, pp.1-49, 2015.

[20] A. Titov, "Thermal Evolution Process and its Relation to Some Philosophical Problems of Physics." Proceedings of the 2nd International Conference on Fluid Flow, Heat and Mass Transfer, pp.153-1 - 153-9, Ottawa, Ontario, Canada, 2015.

[21] A. Titov, "Synchronous Differential Thermal Measurements and New Concept of Temperature." Proceedings of the 2nd International Conference on Fluid Flow, Heat and Mass Transfer, pp.152-1 - 152-9, Ottawa, Ontario, Canada, 2015.

[22] A. Titov, I. Malinovsky, "High-precision temperature measurements of material artifacts." XVIII IMEKO WORLD CONGRESS, "Metrology for a Sustainable Development", , Rio de Janeiro, Brazil, pp.1-7, 2006.

[23] A. Titov, I. Malinovsky, "Precision measurement of self-heating effect of resistance thermometers installed on material artifacrs." XVIII IMEKO WORLD CONGRESS, "Metrology for a Sustainable Development", Rio de Janeiro, Brazil, pp.1-7, 2006.

[24] R. Loudon, L. Allen and D. F. Nelson, "Propagation of electromagnetic energy and momentum through an absorbing dielectric", Phys. Rev. E, vol. 55, pp.1071-1085, 1997.

[25] D. J. Griffiths, "Introduction to Electrodynamics", 3-d ed, Prentice Hall, 1999.

[26] D. V. Sivukhin, "Electricity." in General Course of Physics, 4-th ed, V.3, Physmatlit, Moscow, 2008, pp. 337-355.

[27] D. V. Sivukhin, "Thermodynamics." in General Course of Physics, 4-th ed, V.2, Physmatlit, Moscow, 2008, pp. 162-168.

[28] B. Guinot, "Solar time, legal time, time in use." Metrologia, V.48, pp. S181-S185, 2011

[29] Ch. Darwin and A. R. Wallace, "The Tendencies of Species to Form Varieties, and the Perpetuation of Varieties and Species by Natural Means of Selection." Journal of the Proceedings of Linnean Society of London, Zoology 3, 3 (9), pp.46-50, 1858.

[30] G. Smoot, M. Gorenstein, and R. A. Muller, Phys. Rev. Let. V. 39, pp. 898-903, 1977.

[31] J. V. Nickolas and D. R. White, "Traceable Temperatures", (2nd ed.), J. Willey \& Sons, p.107, 2004.

[32] T. Thornton, J. Marion, "Classical Mechanics of Particles and Systems" (5-th ed.) Thomson, Brooks/Cole, New York - Toronto, p.51, 2004.

[33] R. Penrose, "Gravity and State Vector Reduction", in Quantum Concepts in Space and Time, R. Penrose \& C.J. Isham (eds.), Clarendon Press, Oxford, p.129, 1986.

[34] D. Kleppner, R. J. Kolenkow, “An Introduction to Mechanics", Cambridge University Press, New-York, 2010.

[35] A. Zeilinger, "On the Interpretation and Philosophical Foundation of Quantum Mechanics", in: "Vastakohtien todellisuus", U. Ketvel et al. (Eds.), Helsinki University Press, 1996.

[36] J. D. Jackson, "Classical Electrodynamics", J. Willey and Sons, 3-ed, p. 14, 1999.

[37] W. Pauli's letter to M. Fierz of 13.10.1951. K.V. Laurikainen,

“Wolfgang Pauli and Philosophy" Gesnerus 41, pp. 225-227, 1984

[38] E. Noether, "Invariante Variationsprobleme." Nachr. v. d. Ges. d. Wiss. zu Göttingen, pp. 235-57, 1918.

[39] V. Gerginov, et al, "Uncertainty evaluation of the caesium fountain standard PTB-CSF22", Metrologia,V.47, pp. 65-79, 2010.

[40] M. A. Lombardi, T. P. Heavner and S. R. Jefferts, "NIST Primary Frequency Standards and the Realization of the SI Second", Measure, V.2, No.4, pp.74-89, 2007.

[41] D. W. Allan, "Statistics of atomic frequency standards", Proc. IEEE, V.54, pp 207-220, 1966.

[42] J. C. Hafele and R. E. Keating, "Around - the - World Atomic Clocks: Observed Relativistic Time Gains", Science, V.177, p. 162, 1972.

[43] D. V. Sivukhin, "Optics" in General Course of Physics, 4-th ed, V.5, Physmatlit, Moscow, 2008, pp. 650-705s. 
[44] L. Schaltzer, "The Speed of Light - A Limit on Principle?" aether.lbl.gov/www/classes/p139/speed/space-time.html (1997).

[45] B. Nodland, and J. P. Ralston, "Indication of Anisotropy in

Electromagnetic Propagation over Cosmological Distances", Phys. Rev.

Letts., V. 78, No. 16, pp. 3043-3046, 1997.

[46] S. Chu, and S. Wong, "Linear Pulse Propagation in an Absorbing

Medium", Phys. Rev. Letts, V.48, No. 11, pp. 738-741, 1982.

[47] C. G. B. Garrett and D. E. McCumber, "Propagation of a Gaussian Light

Pulse through an Anomalous Dispersion Medium", Phys. Rev. A 1, p. 305,

1970

[48] B. Hensen, et al, "Experimental loophole-free violation of a Bell

inequality using entangled electron spins separated by $1.3 \mathrm{~km}$ ",

arXiv:1508.05949 [quant-ph], 2015.

[49] P. Kusch, "Observation of the multiple quanta transitions", Phys. Rev. V. 93,

pp. $1022-1034,1954$

[50] A. Einstein's letter to Schrödinger of 22.12.1950 in "Briefe zur

Wellenmechanik”, K. Przibram (ed.), Springer-Verlag, Vienna, pp.36-37,

1963. 